This is the author's copy of the publication as archived with the DLR's electronic library at http://elib.dlr.de. Please consult the original publication for citation.

\title{
Modeling of geared positioning systems: An object- oriented gear contact model with validation
}

van der Linden, FLJ

In aerospace positioning actuators, gear efficiencies of $85 \%$ as well as breakout forces as high as $50 \%$ of the stall load of the actuator are observed at very low temperature conditions. Due to the low efficiency and high loading, stiction in these actuators is common which can lead to limit cycles or problems with controlling the actuators. To be able to correctly predict and assess these effects using simulations, a complete actuator, including motor, inverter, load and controller is needed. This article presents an object based, numerically efficient gear contact in a planar environment with user-defined friction- and stiffness laws. The emphasis of the modeling is not a fully detailed contact model, but the description of a gear contact model which can be used for system simulations like complete aircraft electro-mechanical actuators including control surfaces. The presented model is suitable for complex gearing configurations (e.g. compound planetary gears). This is enabled by breaking down the transmission into the basic gear contacts. By adding masses and constraints from an existing component library, a complete transmission can be modeled. The generated model can be used for standalone simulations or can be used in multidomain simulations like actuator modeling, in order to analyze the complete actuator model including parts such as drives, controllers and mechanical systems. The presented models have been validated using a gear test rig for a single stage spur gear.

\section{Citation Notice}

[1] FLJ van der Linden. Modeling of geared positioning systems: An object-oriented gear contact model with validation. Proceedings of the Institution of Mechanical Engineers, Part C: Journal of Mechanical Engineering Science, June 2015. doi:10.1177/0954406215592056.

Qarticle\{Linden2015GearModeling,

abstract $=\{$ In aerospace positioning actuators, gear efficiencies of 85 \author $=\{$ van der Linden, FLJ $\}$,

doi $=\{10.1177 / 0954406215592056\}$

$\mathrm{file}=\left\{: C \$ \backslash\right.$ backslash\$:/Users/lind $\_$fr $/$AppData/Local/Mendeley Ltd. /Mendeley Desktop/Downloaded/van der Linden - $2015-$ Modeling and validation of gear contacts in an object oriente framework for positioning applications.pdf:pdf\},

$\operatorname{ssn}=\{0954-4062\}$,

journal $=$ \{Proceedings of the Institution of Mechanical Engineers, Part C: Journal of Mechanical Engineering Science $\}$,

month $=j$ un
title $=\{$ Model

(Modeling of geared positioning systems: An object-oriented gear contact model with validation\}\}, 


\section{Modeling of geared positioning systems: An object oriented gear contact model with validation.}

Proc IMechE Part C: J Mechanical Engineering Scienc $0(0): 1-16$

(C)The Author(s) 2015

Reprints and permission:

sagepub.co.uk/journalsPermissions.nav

DOI: 10.1177/0954406215592056

www.sagepub.com/

(3)AGE

\author{
Franciscus L.J. van der Linden
}

\begin{abstract}
In aerospace positioning actuators, gear efficiencies of $85 \%$ as well as breakout forces as high as $50 \%$ of the stall load of the actuator are observed at very low temperature conditions. Due to the low efficiency and high loading, stiction in these actuators is common which can lead to limit cycles or problems with controlling the actuators. To be able to correctly predict and assess these effects using simulations, a complete actuator, including motor, inverter, load and controller is needed. This article presents an object based, numerically efficient gear contact in a planar environment with user-defined friction- and stiffness laws. The emphasis of the modeling is not a fully detailed contact model, but the description of a gear contact model which can be used for system simulations like complete aircraft electromechanical actuators including control surfaces. The presented model is suitable for complex gearing configurations (e.g. compound planetary gears). This is enabled by breaking down the transmission into the basic gear contacts. By adding masses and constraints from an existing component library, a complete transmission can be modeled. The generated model can be used for standalone simulations or can be used in multi-domain simulations like actuator modeling, in order to analyze the complete actuator model including parts such as drives, controllers and mechanical systems. The presented models have been validated using a gear test rig for a single stage spur gear.
\end{abstract}

\title{
Keywords
}

Gear Dynamics, Dynamic Modelling, Multibody Dynamics, Actuators, Powertrain, Experimental Mechanics

\section{Introduction}

When designing an electromechanical actuator at system level, modeling of the system helps the developers predict the performance of the complete actuator. This avoids costly prototypes, helps to speed up the design process and also enables to give a prediction of the design parameters such as forces, moments and currents. Modeling a geared electromechanical aircraft actuator with a compound planetary gear requires a multi-domain approach combining the electrical, mechanical and thermal domain. Furthermore the controller must be simulated to model the complete actuator. In aerospace actuator gears at $-55^{\circ} \mathrm{C}$, the transmission efficiencies are usually very low. Internal studies at several aircraft part manufacturers have shown that efficiencies around $85 \%$ and breakout forces of $50 \%$ of the stall load are not uncommon due to the extreme temperature conditions. Combining these high gear losses with high preloading, limit cycles and hunting during positioning tasks are challenges of actuator designers.

During the design process of such an actuator, typical questions arise when modeling the gearbox such as: How can the computational effort of the actuator simulation for optimization runs be kept low? What is the frequency response of the actuator? What is the overall efficiency of the system at different load levels? Do the sticking forces in combination with the controller lead to hunting or limit cycles?

Furthermore, the best modeling detail level for a simulation is often not known and might change during the design process. Therefore, the organization and availability of the gear modeling knowledge is of high importance to support the model designer in the design process. Since the computational effort of these multi-domain systems quickly increases, it is important that the gear models are numerically efficient. For such simulations, often used approaches based on FEM calculations or simplified 2D contact algorithms are in most cases prohibitively expensive whereas 1D rotational effects are in some cases not accurate enough. This is not only the case for electromechanical actuators, but also applies to models like wind turbines and car transmissions.

The goal of this work is to combine the available gear knowledge and to implement a set of object-oriented models that deliver good simulation accuracy paired with computational efficiency and modularity. At the same time, the versatility of the gear models must be kept high. In this work, this is achieved by analyzing the gear forces $1 ; 2$ and the introduction of an offline calculation of friction parameters based on the gear geometry. The models are implemented in the object-oriented Modelica language, which makes the knowledge readily available to the model designer. This enables carrying out integrated system simulations using existing Modelica models like motors, kinematics or brakes by interconnecting them with the presented gearbox model.

German Aerospace Center - DLR

\section{Corresponding author:}

German Aerospace Center - DLR Oberpfaffenhofen, 82234 Wessling, Germany

Email: franciscus.vanderlinden@dlr.de 
A set of gear models was implemented with different levels of detail. These models can be selected depending on the given task: An ideal gear contact (without elasticity or friction), an elastic model with an elastic gear contact but without friction losses from previous work ${ }^{3}$, and finally a combined lossy-elastic model including elasticity and friction effects. The friction as well as the elasticity is modularly implemented for full flexibility. Currently Coulomb friction ${ }^{2}$ as well as velocity dependent friction models ${ }^{1 ; 4}$ are implemented. Moreover, friction excitations can be analyzed $^{5-11}$ by using a position dependent friction. Concerning elasticity, several elastic models are implemented: a simple spring-damper, a nonlinear springdamper ${ }^{1}$, a backlash model and a position dependent springdamper to model forcing errors ${ }^{12 ; 13}$.

Stick-slip behavior ${ }^{14 ; 15}$ is important for (preloaded) positioning applications. Next to this, stiction can sometimes be used to aid holding a load. Therefore, in this work, the stiction of the gear at zero velocity is explicitly handled using a state machine. This ensures that stiction and stickslip is treated in a numerically efficient, consistent way with a nonzero friction force at zero velocity. A state machine is used here to avoid problems with position creepage under load and makes it possible to correctly simulate motor holding torques under load.

Bearing- and windage-losses are omitted as they are outside the direct gear contact, which is described in this paper. Using external components, these effects can be readily taken into account.

Due to the modularity of the system, it is possible to extend the model during the development of the actuator and increase the model complexity when needed or when more detailed information becomes available.

\section{Literature Overview}

The works from Buckingham ${ }^{2}$ as well as Niemann and Winter ${ }^{1}$ highlight the fundamental properties of gear connections. Since in these works many gear properties are analytically derived, they are of high interest for numerically efficient gear modes. For gear dynamics, there are two major model review papers: Özgüven and Houser ${ }^{16}$ and Parey and Tandon ${ }^{17}$ give a valuable overview of the research efforts till 2003.

More recently, the emphasis of many works is focused on gears with varying stiffness of the gear mesh and bearing influences: Guo and Parker ${ }^{18}$ made an extensive analysis of a planetary gear system with bearing clearance. Parker and $\mathrm{Wu}$ did a similar analysis ${ }^{19}$, where they are using a flexible ring. Ottewill et al. ${ }^{13}$ investigated gear rattle and the interaction between eccentricities and manufacturing errors. They measured the errors in a gearbox and included backlash in their models, however, no friction is included. Also Pedersen et al. ${ }^{20}$ evaluated stiffness variations in their analysis. They used a commercial computer program to calculate the stiffness and used this as an input for their own frequency analysis. Similar gear stiffness analysis using FEM have been made by Kiekbusch et al. ${ }^{21}$. A good overview of friction modeling in gearboxes is given by Vinayak and Singh ${ }^{22}$. They compare several modeling strategies for gear friction. Pelchen et al. ${ }^{23}$ made a non elastic rotational model with friction based on efficiency in Modelica which can handle stiction effects.

Elasticity and friction models are also combined: Velex et al. ${ }^{6-9}$ and De Gevigney et al. ${ }^{24}$ have worked on mounting errors, profile errors and friction induced excitations. They both use an approach in which the contact between the gear wheels is evaluated at all possible contact positions. Using this approach, they are able to simulate wide ranges of gear imperfections. Kar and Mohanty ${ }^{5}$ did a similar analysis where also the influence of missing teeth is analyzed. Vinayak et al. ${ }^{10 ; 11}$ have made a thorough analysis of a $6 \mathrm{DOF}$ gear system with 6DOF stiffness analysis. With the approaches of Velex et al. and Vinayak et al. as well as Kar and Mohanty, the simulation accuracy will be between fully elastic FEM models and 1D rotational- or planar approaches. In their works, no special stiction handing algorithms are mentioned. Also commercial programs like SIMPACK have implemented gear contact algorithms. Ebrahimi and Eberhard $^{25}$ use a 2D contact searching algorithm with rigid, but tangentially flexible teeth. They use a contact finding algorithm to allow for multiple contacts.

On the other hand, there are studies that are driven by practical problems: Ahmed et al. ${ }^{14}$ studied preloaded gear drives. They mention that in the positioning control of these drives, stiction behavior of the gears becomes problematic (Stick-slip and hunting problems). Also Garcia et al. ${ }^{15}$ concludes that the oscillations by the friction at very low speed caused by stiction are large.

\section{Problem description}

To be able to describe the gear contact with sufficient detail for the development of (positioning) control- and health monitoring algorithms, following requirements must be met:

- Integration of the gear model into complete system models for control and health monitoring design,

- Modularity to enable different friction laws as well as different stiffness models,

- Fast simulation performance to support optimization runs using a complete system model,

- Operation in all quadrants since the power flow is often reversed in positioning applications,

- Sticking of the gear: The breakout force/ torque at zero speed must be able to accommodate non steady friction coefficients to deal with static/ dynamic friction coefficients.

Since the model performance must be high enough for complete system simulations and optimization runs, contact searching algorithms (e.g. Ebrahimi and Eberhard ${ }^{25}$ ) are prohibitively expensive. By avoiding these contact searching algorithms, the deformations of bearings, axis and teeth cannot be taken into account for the calculation of the gear forces. Instead of finding the contacts positions, the gear forces must be estimated using average friction forces. To be able to model position dependent effects caused by the tooth contact, pre-calculated values can be used.

In this work, a new modeling level is presented. It is aiming at enabling the system engineer to model gear components with just enough details to allow accurate 


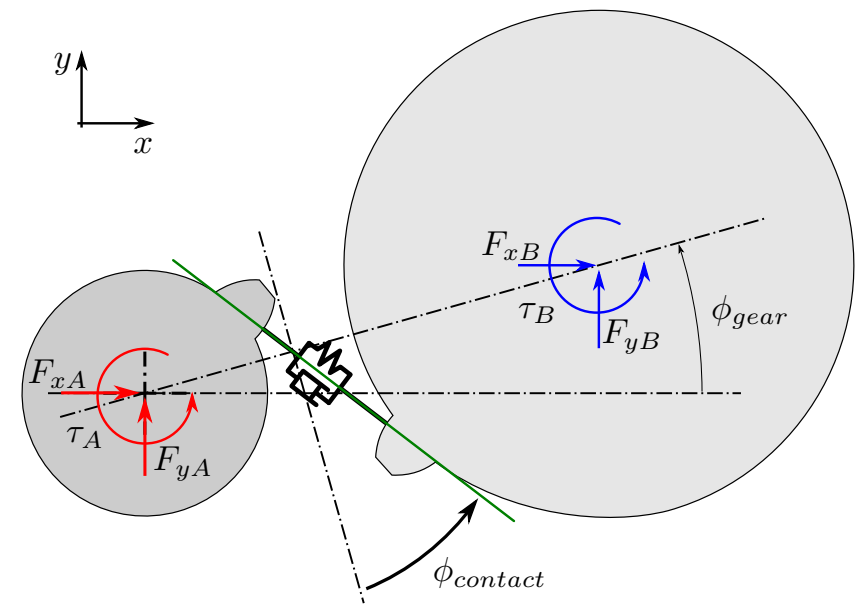

Figure 1. Basic gear forces on the contact of a single tooth.

system level analysis and design. In this paper, the approach to reach this modeling level is outlined.

The specificities of our approach is outlined next.

\section{Gear Forces}

The forces acting on the rotational axis of a spur gear set are shown in Figure 1. These forces and torques are needed for a full planar description of the two gear wheels and will finally be used for the interface to the planar multi-body Modelica environment. For the calculation of these forces, the individual tooth forces are analyzed and averaged over the contact length. This enables fast calculations by precalculating the contact parameters of the tooth. By using a position dependent averaging, it is possible to include the effects of multiple tooth contacts.

\section{Gear contact description}

The gear contact, analyzed in this paper, is considered to be an involute gear contact since this is the most used gear connection in engineering applications. In Figure 2, a schematic overview of two gear wheels in contact is shown as is commonly used in literature e.g. ${ }^{1 ; 2 ; 4}$. In this Figure, the contact point $C$ moves along the green line of action. The position of the contact point on this line is given by $d_{A}$ (and consequently $d_{B}$ ). The gear ratio is defined by $\frac{r_{A}}{r_{B}}=\frac{l_{A}}{l_{B}}=i$. This ratio is constant for each gear contact point. $d_{A}, d_{B}, r_{A}$ and $r_{B}$ are defined in Figure 2. The contact angle $\phi_{\text {contact }}$ defines the orientation of the line of action. This contact angle is dependent on the loading direction; in the case where gear $A$ drives gear $B$ the $\phi_{\text {contact }}$ is as displayed in the Figures 2 and 3 , in the opposite case where $B$ drives $A$, the other side of the gear teeth are in contact and therefore $\phi_{\text {contact }}$ will be negative.

Figure 3 shows a free body diagram of the two gears in contact. The forces of only one contact point are displayed. Using Figure 3, it is possible to create the torque and force balances of each gear wheel. These forces and torques are resolved in the fixed coordinate system shown in this Figure. Instead of using a gear-fixed coordinate system, a fixed coordinate system and variable gear angle $\phi_{\text {gear }}$ is used, which defines the rotation of the gear system with respect to the reference frame. This makes it possible to use the contact model also in more complex gear systems with multiple gear contacts (e.g. all kinds of Epicyclic gearing configurations). The resulting forces for this gear contact are:

$$
\begin{aligned}
\tau_{A}= & F_{n} l_{A}-F_{t} d_{A}, \\
\tau_{B}= & F_{n} l_{B}-F_{t} d_{B}, \\
F_{x A}= & -F_{n} \sin \left(\phi_{\text {gear }}-\phi_{\text {contact }}\right)- \\
& F_{t} \cos \left(\phi_{\text {gear }}-\phi_{\text {contact }}\right), \\
F_{y A}= & F_{n} \cos \left(\phi_{\text {gear }}-\phi_{\text {contact }}\right)- \\
& F_{t} \sin \left(\phi_{\text {gear }}-\phi_{\text {contact }}\right) \\
F_{x B}= & -F_{x A} \\
F_{y B}= & -F_{y A} .
\end{aligned}
$$

Note that the lengths $d_{A}$ and $d_{B}$ are changing depending on the position of the contact on the line of action.

\section{Introduction of Mesh Distance, Mesh Velocity and Mesh Deformation}

For the calculation of the (position dependent) gear forces and friction, the mesh position $x_{m e s h}$, the mesh deformation $\Delta_{A B}$ and the mesh velocity $v_{m e s h}$ will be needed in following Sections. In this Section, these terms will be introduced.

To keep track of how the gear wheels move with respect to each other, the mesh distance $x_{m e s h}$ is introduced. The distance $x_{m e s h}$ can be calculated for both gear wheels and is defined as the distance the gear wheels have traveled through the meshing point $P$ (see Figure 2 for reference on the definition of the variables). The total meshed distance can be calculated for both gearwheels:

$$
\begin{aligned}
& x_{\text {mesh }, A}=\phi_{A} r_{A}-\phi_{\text {gear }} r_{A}, \\
& x_{m e s h, B}=-\phi_{B} r_{B}+\phi_{g e a r} r_{B} .
\end{aligned}
$$

In these equations, $r_{A}$ and $r_{B}$ are the pitch radii from gear $A$ and $B$. From Equations (7) and (8) it becomes clear that the mesh distance $\left(x_{m e s h, A}\right.$ or $\left.x_{m e s h, B}\right)$ can be constant although the gear wheels are rotating. This is the case if $\phi_{A}=\phi_{\text {gear }}$ or $\phi_{B}=\phi_{\text {gear }}$. This is not only a theoretical implication; in a Ravigneaux gearset used as differential for example this is often the case. Note that the gear axis have to be constraint on a distance $r_{A}+r_{B}$ as a relative motion will alter $x_{m e s h, A}$ and $x_{m e s h, B}$.

The mesh deformation represents the difference between the mesh positions. It is defined by the deformation of the gear contact including tooth bending:

$$
\Delta_{A B}=x_{m e s h, A}-x_{m e s h, B} .
$$

For position dependent stiffness and friction effects, the average mesh position is needed. It is assumed that the deformation of the gear wheels is equal; Gears with higher number of teeth have a relatively high tooth bending, which is compensated by the stiff tooth contact (due to the low curvature). For gears with lower number of tooth, the effect is the other way around: The low tooth bending is compensated by a high contact stiffness. The mesh position is defined as:

$$
x_{m e s h}=\frac{x_{m e s h, A}+x_{m e s h, B}}{2} .
$$




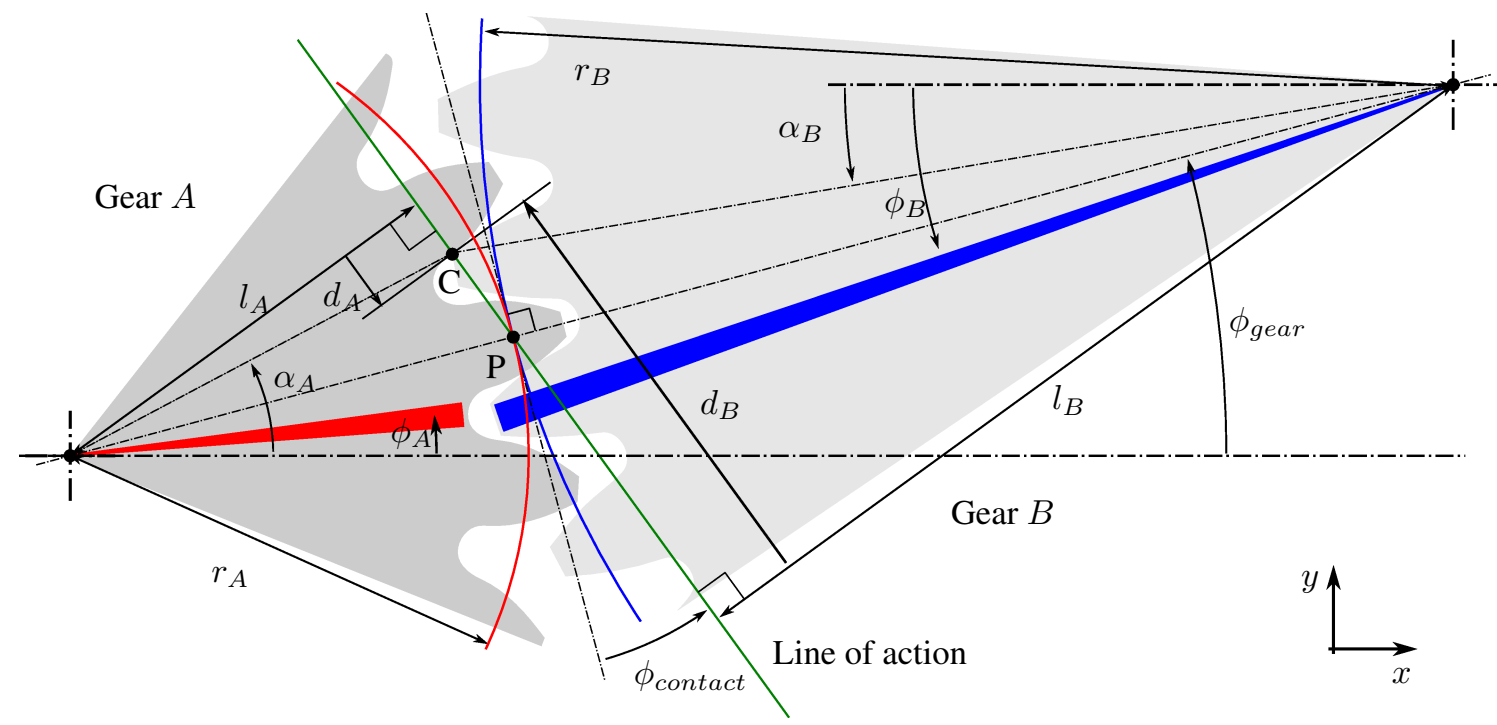

Figure 2. Schematic overview of two involute gears in contact. The blue and red triangles are fixed markers on the gear wheels. In the figure $\omega_{A}<0$ and Gear $A$ drives gear $B$.

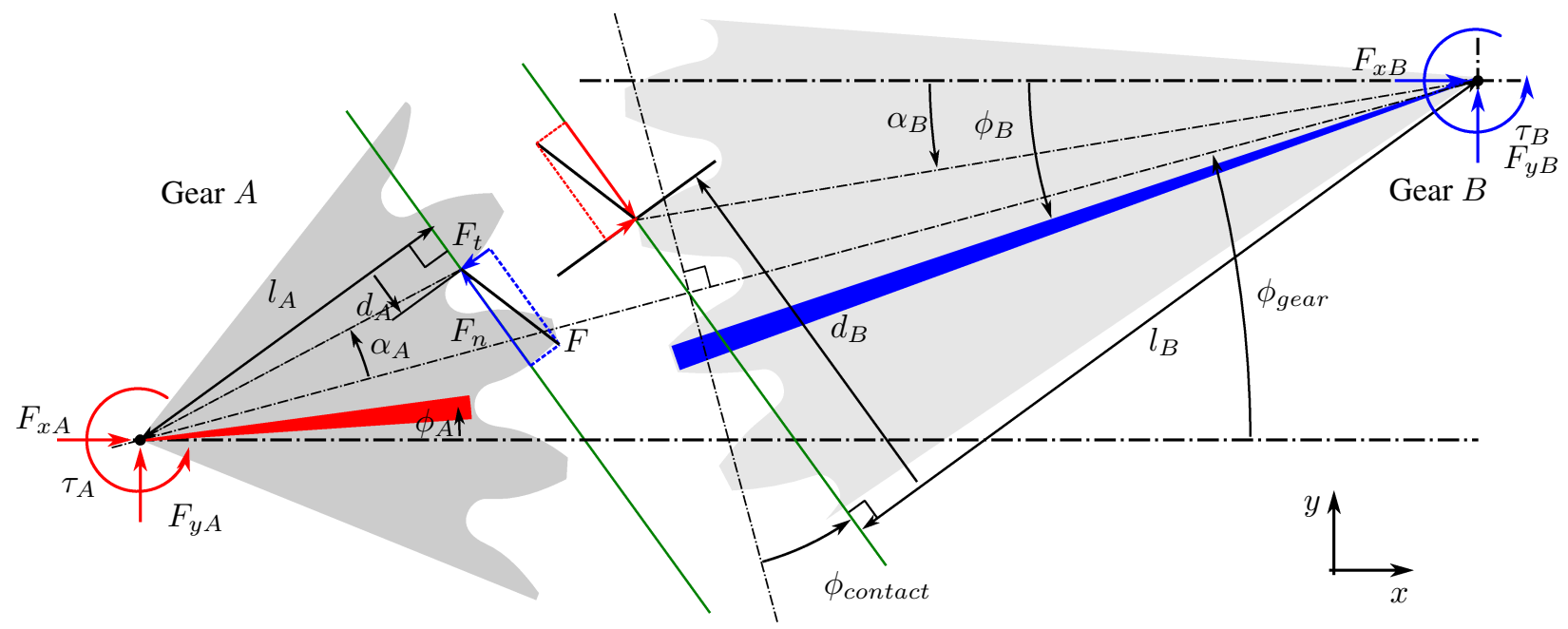

Figure 3. Free body diagram of two involute gears. In the figure $\omega_{A}<0$ and Gear $A$ drives gear $B$.

The average mesh velocity is given by differentiation of the average mesh position (10) with respect to time. This velocity represents the feed velocity of the gears through the meshing point $P$.

$$
v_{m e s h}=\frac{\dot{x}_{m e s h, A}+\dot{x}_{m e s h, B}}{2} .
$$

\section{Friction Force}

The friction force $F_{t}$ is dependent on the normal force $F_{n}$ and the sliding speed $v_{s}$ between the gears. To calculate the sliding speed, the gear contact is examined: In Figure 4 and 5, the gear contact in two different loading conditions is shown. In these Figures, $T_{1}$ and $T_{2}$ are the contact points of the line of action and the base circle of gear $A$ respectively $B$. The mesh contact is from point $A$ to $B$. Point $A$ is the first contact point of the mesh and is defined by the intersection between the line of action and the addendum circle of gear $B$. Point $B$ is the last point of contact and is defined by the intersection from the line of action with the addendum circle of gear $A$. The meshing point $P$ is the intersection of the line $O_{A} O_{B}$ and the line of action. It is also the position where the pitch circles of gear $A$ and $B$ are touching.

The tangential velocities of the gears $A$ and $B\left(v_{t, A} \&\right.$ $\left.v_{t, B}\right)$ are defined as the velocity of each gear at the contact point $C$ perpendicular to the line of action. These velocities in the tangential direction are obtained by a multiplication of the rotational speed $\left(\omega_{A}, \omega_{B}\right)$ by the arm perpendicular on the tangential velocity $\left(d_{A}, d_{B}\right)$.

$$
\begin{aligned}
v_{t, A} & =\omega_{A} d_{A}, \\
v_{t, B} & =\omega_{B} d_{B} .
\end{aligned}
$$

Where is used: $\omega_{A}=\dot{\phi}_{A}$ and $\omega_{B}=\dot{\phi}_{B}$.

In Figure 4, the tangential velocity for each possible gear position is highlighted for the indicated case. The red triangle is the tangential velocity of Gear $A$, the blue triangle the tangential velocity of Gear $B$. The magnitude of the tangential velocity is shown by the size of the triangle. The relative tangential velocity $v_{s}$ between gear $A$ and $B$ is the sliding velocity which occurs in the contact point. A more detailed description can be found in gear design books 
e.g. ${ }^{1 ; 2}$.

$$
\begin{aligned}
v_{s} & =v_{t, A}-v_{t, B}, \\
& =\omega_{A} d_{A}-\omega_{B} d_{B} .
\end{aligned}
$$

Note that at the pitch point $P$, the relative tangential velocity is zero as expected (pure rolling at the pitch circle).

The friction force $F_{t}$ is defined using the speed dependent friction coefficient $\mu\left(v_{s}\right)$, which can also be written as $\mu\left(v_{m e s h}, d_{A}\right)$ since the sliding velocity is dependent of the total mesh speed speed $v_{m e s h}$ and the distance $d_{A}$ (see Figure 4). This yields:

$$
F_{t}=\left\{\begin{array}{l}
\text { if } v_{\text {mesh }} \neq 0 \text { or }\left|F_{t}\right| \geq\left|\mu\left(v_{\text {mesh }}, d_{A}\right) F_{n}\right|: \\
s_{v}\left(v_{\text {mesh }}, d_{A}\right) \mu\left(v_{\text {mesh }}, d_{A}\right)\left|F_{n}\right| \\
\text { if } v_{\text {mesh }}=0 \text { and }\left|F_{t}\right|<\left|\mu\left(v_{\text {mesh }}=0, d_{A}\right) F_{n}\right|: \\
\quad \text { so that } v_{\text {mesh }}=0
\end{array}\right.
$$

In Equation (15), $s_{v}$ is the sign of the sliding velocity $v_{s}$. The normal force $F_{n}$ must be absolute since it does not matter which gear face is pressed on the other. The second part of the equation deals with the results in the case of $v_{\text {mesh }}=0$. In this case, the friction force $F_{t}$ is calculated as the force to keep the mesh velocity $v_{\text {mesh }}$ zero. This is the case as long as $\left|F_{t}\right|<\left|\mu\left(v_{m e s h}=0, d_{A}\right) F_{n}\right|$, as this is the upper bound of the friction at zero mesh velocity.

In Figure 5, a different loading condition is shown. In this loading condition, the line of action is flipped and therefore the contact points are different. Using the same analysis, it is possible to define $s_{v}\left(v_{m e s h}, d_{A}\right)$ also for the load-case shown in Figure 5. To deal with the other two gear loading possibilities, the gear velocities must be inverted. This inversion leads then directly to the inversion of $s_{v}\left(v_{m e s h}, d_{A}\right)$. Using this property, it is possible to create an overview of all possible conditions. This overview is given in Table 1 . In this table, $s_{v, A P}$ is the sign of the sliding velocity $s_{v}\left(v_{m e s h}, d_{A}\right)$ between point $A$ and point $P, s_{v, P B}$ between $P$ and $B$.

\section{Individual Gear Tooth Forces reduction to overall Gear Forces}

In the previous section, all gear forces are defined for a single tooth contact of a spur gear. Using a contact finding algorithm, this could be directly implemented. However, for an efficient simulation of large systems, such contact searching algorithms must be avoided. To avoid these contact searching algorithms, two approaches are possible:

1. Generation of a lookup table which expresses the gear forces $\tau_{A}, \tau_{B}$ and $F_{t}$ as function of $F_{n}$, $\phi_{\text {gear }}-\phi_{\text {contact }}$ and the exact gear geometry.

2. Construction of an analytical function which approximates the gear forces based on averaging.

To generate an accurate lookup table, the gear geometry and properties must be precisely known to cover all of its effects. Including the influence of deforming teeth on the gear properties, the gear contacts must be assessed for all operating conditions using specialized programs e.g. ${ }^{26}$. For the study of broken parts, pre-design or the generation of detailed gear specifications, this approach cannot be used as the design of the gear is in most cases not known. In this case, an approximation must be made based on available knowledge. This approximation should be able to include the effects of gear meshing, without modeling the individual contact.

On the other hand, it is possible by averaging the friction forces to obtain an analytical function expressing the friction forces on the gear. This approach makes it possible to obtain a sufficiently detailed model without an accurate knowledge of the system geometry, which makes it suitable for pre-design stages and cases where the exact geometry is not known. To obtain the analytical function, several assumptions have to be made.

Anderson and Loewenthal ${ }^{27}$ calculate the average power loss over a tooth contact to calculate gearbox efficiencies. To do so, the integral of the power losses over the contact length is divided by the contact length. To calculate the mean friction forces, a similar approach is used in this paper. The average friction force is obtained by integrating the friction forces over the contact length and dividing by the integral of the contact position (total contact length)

$$
\bar{F}_{t}=\frac{\int_{A}^{B} F_{t}\left(d_{A}\right) \mathrm{d} d d_{A}}{\int_{A}^{B} \mathrm{~d} d_{A}}
$$

To calculate the average friction force, the integral must be split in multiple parts to take the discontinuity of the normal force over the contact length into account, which is caused by different contact ratios *. The varying normal force and tooth contacts are shown in Figure 6. For a tooth ratio between 1 and two, the gear contacts points vary between one and two. When a constant torque is given to the gear wheels, the tooth load for a single contact is double the load as for a double contact, since the load is shared between both contact points. Assuming a contact ratio between one and two, the integral must be split into three parts to take the discontinuities of the normal force into account:

$$
\bar{F}_{t}=\frac{\int_{A}^{X 1} F_{t}\left(d_{A}\right) \mathrm{d} d_{A}}{\int_{A}^{X 1} \mathrm{~d} d_{A}}+\frac{\int_{X 1}^{X 2} F_{t}\left(d_{A}\right) \mathrm{d} d_{A}}{\int_{X 1}^{X 2} \mathrm{~d} d_{A}}+\frac{\int_{X 2}^{B} F_{t}\left(d_{A}\right) \mathrm{d} d_{A}}{\int_{X 2}^{B} \mathrm{~d} d_{A}} .
$$

It is possible to solve Equation (17) now for the different parts of the contact on the line of action to obtain the average friction force. This can be done using a pre-processing method with a detailed contact description. The results can be stored in a lookup table as suggested before. However, in order to be concise, two assumptions will be made:

\footnotetext{
* The contact ratio is a measure of the average number of teeth in contact. A contact ratio of 1.5 would mean that for $50 \%$ of the line of action there is a single tooth contact and for $50 \%$ of the line of action there are two teeth in contact.
} 
Table 1. Sliding velocity of contact point of each loading condition. The quadrant is the operational mode of the gear.

\begin{tabular}{cccccrr}
\hline$v_{\text {mesh }}$ & Power flow & $F_{n}$ & $\phi_{\text {contact }}$ & Quadrant & $s_{v, A P}$ & $s_{v, P B}$ \\
\hline \multirow{2}{*}{$>0$} & $A \rightarrow B$ & $>0$ & $<0$ & 1 & 1 & -1 \\
& $B \rightarrow A$ & $<0$ & $>0$ & 2 & -1 & 1 \\
$<0$ & $B \rightarrow A$ & $>0$ & $<0$ & 3 & -1 & 1 \\
& $A \rightarrow B$ & $<0$ & $>0$ & 4 & 1 & -1 \\
\hline$=0$ & - & - & 0 & 0 & & \\
\hline
\end{tabular}

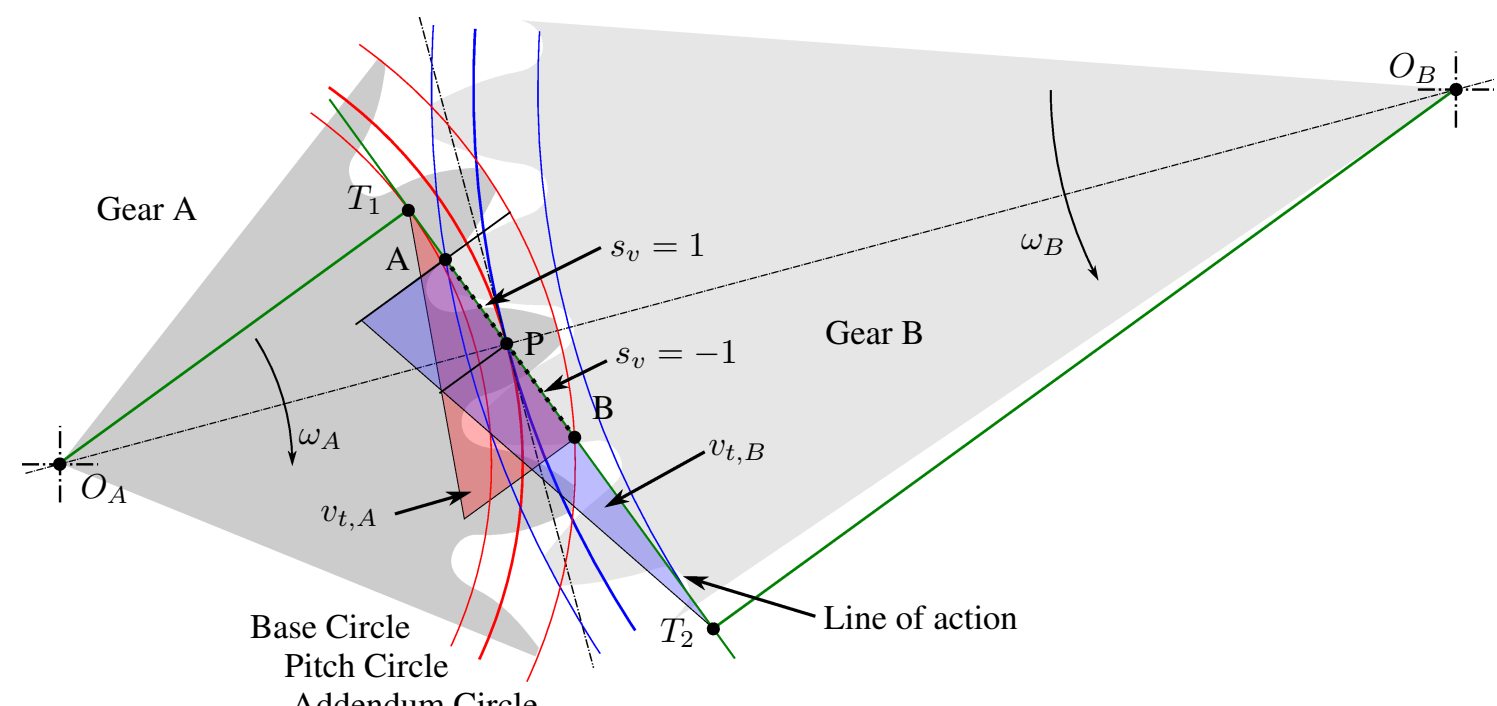

Addendum Circle

Figure 4. Schematic overview of two involute gears in contact operating in quadrant 4: Gear $A$ drives gear $B$, the rotational speed is indicated by the direction of the arrows next to $\omega_{A}$ and $\omega_{B}$ (rotation is clockwise so $\omega_{A}<0 \vee \dot{\phi}_{\text {gear }}=0 \Rightarrow v_{m e s h}<0$ ). The contact angle $\phi_{\text {contact }}$ is positive.

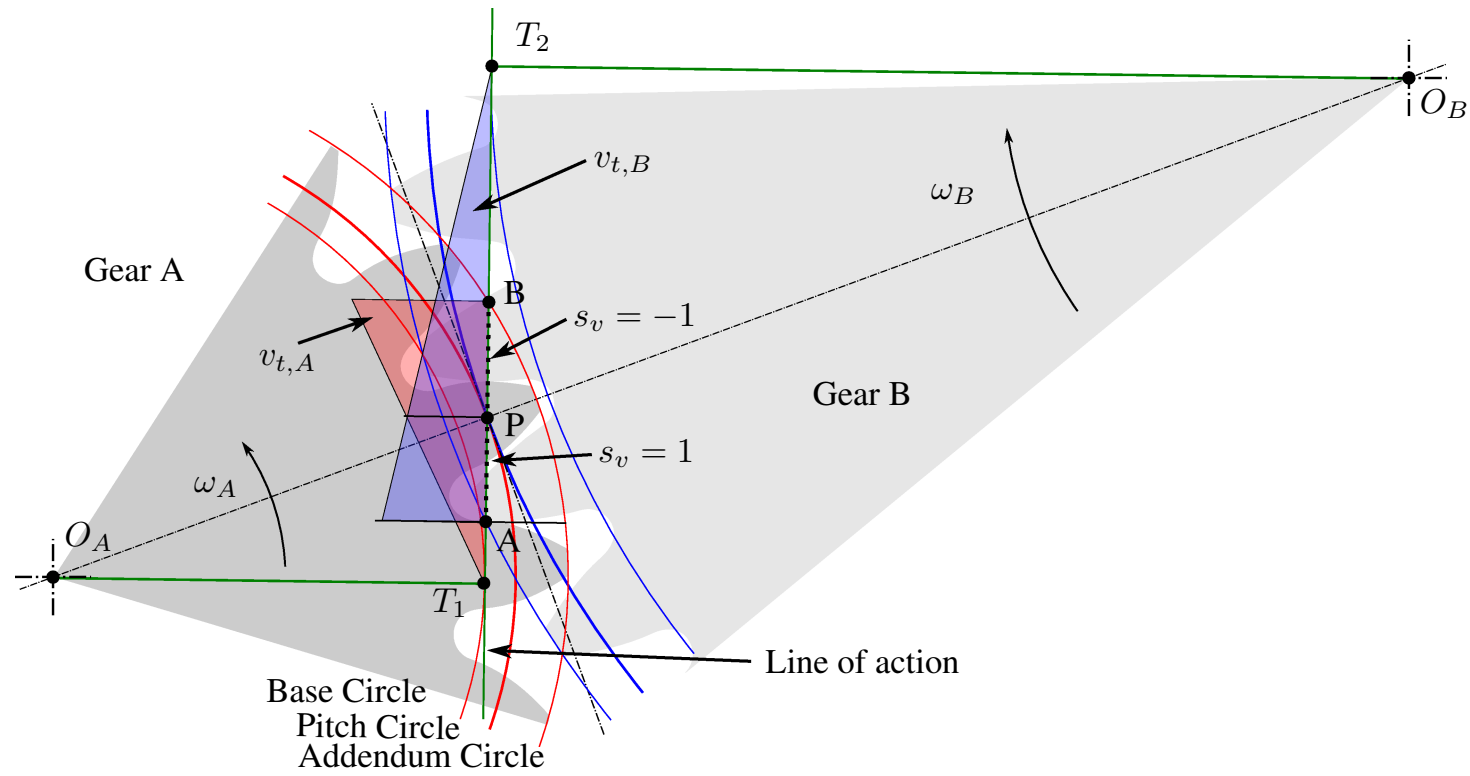

Figure 5. Schematic overview of two involute gears in contact operating in quadrant 1. Gear $A$ drives gear $B$, the rotational speed is indicated by the direction of the arrows next to $\omega_{A}$ and $\omega_{B}$ (rotation is counter clockwise so $\omega_{A}>0 \vee \dot{\phi}_{\text {gear }}=0 \Rightarrow v_{\text {mesh }}>0$ ). The contact angle $\phi_{\text {contact }}$ is negative.

Assumption 1. The normal force $F_{n}$ is constant over the line of action.

The normal force $F_{n}$ is constant over the line of action when the complete line of action from point $A$ to $B$ (see Figure 4$)$ is in contact. For each position on this contact line, a constant width is in contact $\left(\frac{\mathrm{d} \text { facewidth }}{\mathrm{d} d_{\mathrm{A}}}=\right.$ constant $)$. This means that the gear forces are "smeared" over the line of action (From point $A$ to $B$ ) instead of single contact points. This assumption is not only a theoretical assumption, it can be reached by using helical gears with sufficient helix 


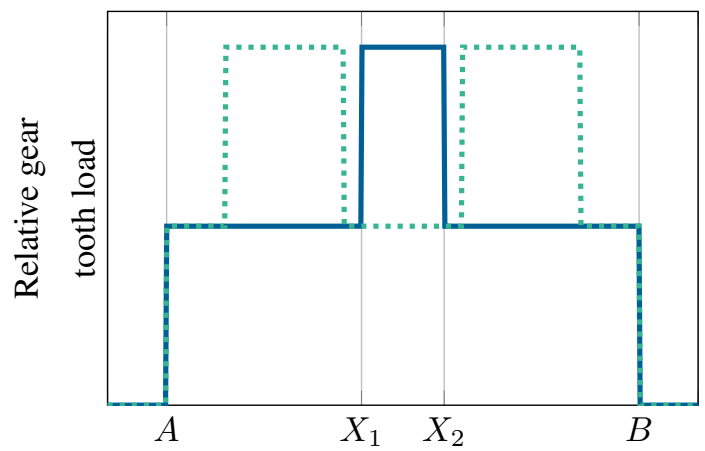

Distance along path of contact

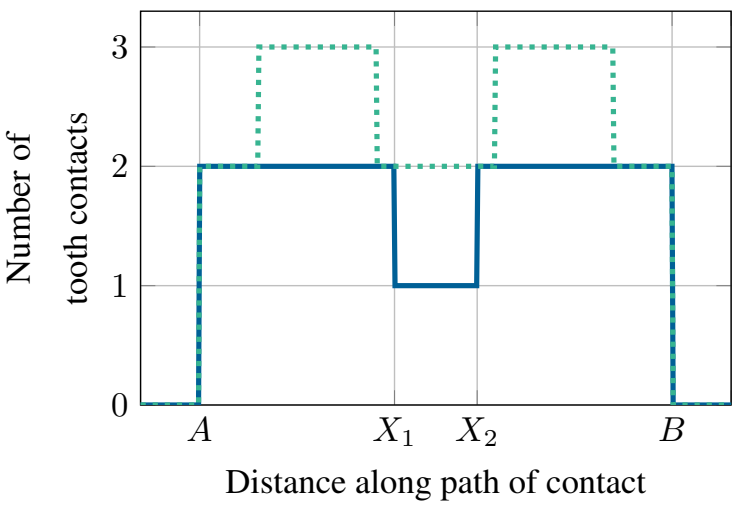

Figure 6. Relative tooth load for contact ratios between 1 and 2 $(-)$ and for a ratio between 2 and $3(n n$.$) . For a contact$ ratio between 1 and 2, there are two contacts between $A$ and $X_{1}$ as well as between $X_{2}$ and $B$. Between $X 1$ and $X 2$ there is only a single tooth contact. The varying number of teeth in contact lead to variable gear tooth loading.

angle and width. Note that this assumption does not limit the global variation of the normal force $F_{n}$, enabling time varying loads. Using Assumption 1, it is possible to derive an average of the contact forces over all possible contact positions. In most cases, only a global coefficient of friction is known, which can be dependent on the mesh velocity. This can be used to further simplify the calculations if the friction coefficient is assumed to be independent of the local sliding velocity on the contact line.

Assumption 2. The friction coefficient is constant on the line of action.

When the friction coefficient is assumed to be constant on the line of action, $\mu\left(v_{\text {mesh }}, d_{A}\right)$ becomes independent of $d_{A}$. Hence it is possible to use $\mu\left(v_{\text {mesh }}\right)$ as the friction coefficient. This causes the gear friction to be only dependent on the sign of the sliding speed $s_{v}$, the normal force $F_{n}$ and the overall gear mesh velocity. Note that this assumes a friction coefficient that is independent on the locally varying sliding velocity on the line of action. An average friction coefficient is therefore used over the complete line of action which is only dependent on the mesh velocity $v_{m e s h}$ as sometimes used for gear design e.g. ${ }^{4}$

Using Assumption 2 is therefore possible to use a global varying friction coefficient which is constant over the gear contact. Using Table 1, it is possible to calculate for each loading condition and speed the mean gear forces and torques. In the sections below, the calculations are made for a single loading condition.
To obtain the moments $\tau_{A}$ and $\tau_{B}$, only the tangential parts of the resultant forces (1) - (2), $\tau_{t, A}=-F_{t} d_{A}$ and $\tau_{t, B}=-F_{t} d_{B}$ have to be analyzed since only $F_{t}$ is changing over the contact position. $\tau_{n, A}=F_{n} l_{A}$ and $\tau_{n, B}=F_{n} l_{B}$ are not dependent on $F_{t}$ or $d_{A}$ and thus do not need averaging.

\section{Average tangential force}

The average friction force is presented in Equation (16). Using Assumption 1, it follows that the integral does not need to be split into multiple parts as done in Equation (17) as the normal force $F_{n}$ is continuous over the contact line. In this Section, the case $F_{n}>0$ and $v_{m e s h}<0$ is worked out. Taking Equation (16) and combining it with Equation (15) gives:

$$
\begin{aligned}
\bar{F}_{t}= & \frac{\int_{A}^{B} F_{t}\left(d_{A}\right) \mathrm{d} d_{A}}{\int_{A}^{B} \mathrm{~d} d_{A}} \\
= & \frac{\int_{A}^{B} s_{v}\left(v_{m e s h}, d_{A}\right) \mu\left(v_{m e s h}, d_{A}\right)\left|F_{n}\left(d_{A}\right)\right| \mathrm{d} d_{A}}{\int_{A}^{B} \mathrm{~d} d_{A}} .
\end{aligned}
$$

Using Assumption 2, it is possible to obtain an integral which is only dependent on the gear geometry. The integral has to be split into 2 parts, because $s_{v}$ is not continuous in $d_{A}$. This yields:

$$
\bar{F}_{t}=\mu\left(v_{m e s h}\right)\left|F_{n}\right| \frac{\int_{A}^{P} s_{v, A P} \mathrm{~d} d_{A}+\int_{P}^{B} s_{v, P B} \mathrm{~d} d_{A}}{\int_{A}^{B} \mathrm{~d} d_{A}}
$$

From Table 1, we see that $s_{v, A P}=-s_{v, P B}$. Substituting this and working out the integral yields:

$$
\begin{aligned}
& \bar{F}_{t}=\mu\left(v_{\text {mesh }}\right)\left|F_{n}\right| s_{v, A P} \frac{\left.d_{A}\right|_{A} ^{P}-\left.d_{A}\right|_{P} ^{B}}{\left.d_{A}\right|_{A} ^{B}}, \\
& \bar{F}_{t}=\mu\left(v_{\text {mesh }}\right)\left|F_{n}\right| s_{v, A P} \frac{2 \overline{T_{1} P}-\overline{T_{1} A}-\overline{T_{1} B}}{\left(\overline{T_{1} B}-\overline{T_{1} A}\right)} .
\end{aligned}
$$

The notation for the distance between points $X$ and $Y$ is defined as $\overline{X Y}$. In Equation (21), the distances $\overline{T_{1} A}, \overline{T_{1} B}$ and $\overline{T_{1} P}$ are constant. To simplify the notation, the new constant $\overline{d_{F}}=\frac{2 \overline{T_{1} P}-\overline{T_{1} A}-\overline{T_{1} B}}{\left(\overline{T_{1} B}-\overline{T_{1} A}\right)}$ is introduced:

$$
\overline{F_{t}}=\overline{d_{F}} s_{v, A P} \mu\left(v_{m e s h}\right)\left|F_{n}\right| .
$$

Analyzing $\overline{d_{F}}$ shows that in the case of two uniform gears in contact, $\overline{d_{F}}$ (and therefore $\bar{F}_{t}$ ) is zero since $2 \overline{T_{1} P}=$ $\overline{T_{1} A}+\overline{T_{1} B}$. This interesting result is caused by the fact that the friction force on both sides from pitch point $P$ (see Figure 4 ) is equal, but inversed since $s_{v}$ changes at the pitch point. 


\section{Derivation of the mean moment}

The mean moments $\overline{\tau_{t, A}}$ and $\overline{\tau_{t, B}}$ can be calculated in the same way as the average friction force $\overline{F_{t}}$. The case $F_{n}>0$ is will be used for the calculations in this section leading to $\tau_{t, A}=-F_{t} d_{A}$. For the case of $F_{n}<0$, the contact line flips as already shown. This leads to a different gear torque: $\tau_{t, A}=F_{t} d_{A}$.

$$
\begin{aligned}
& \text { Calculation of } \overline{\tau_{t, A}} \text { : } \\
& \qquad \begin{aligned}
\overline{\tau_{t, A}} & =\frac{\int_{A}^{B}-F_{t} d_{A} \mathrm{~d} d_{A}}{\int_{A}^{B} \mathrm{~d} d_{A}} \\
& =\frac{\int_{A}^{B}-\mu\left(v_{m e s h}\right) s_{v}\left(v_{m e s h}, d_{A}\right)\left|F_{n}\right| d_{A} \mathrm{~d} d_{A}}{\int_{A}^{B} \mathrm{~d} d_{A}} .
\end{aligned}
\end{aligned}
$$

Since $s_{v}\left(v_{\text {mesh }}\right)$ is discontinuous, the integral is split in two continuous parts, similar to the approach in Equation (19).

$$
\begin{aligned}
\overline{\tau_{t, A}}= & \frac{\int_{A}^{P}-\mu\left(v_{m e s h}\right) s_{v, A P}\left|F_{n}\right| d_{A} \mathrm{~d} d_{A}}{\left.d_{A}\right|_{A} ^{B}}+ \\
& \frac{\int_{P}^{B}-\mu\left(v_{m e s h}\right) s_{v, P B}\left|F_{n}\right| d_{A} \mathrm{~d} d_{A}}{\left.d_{A}\right|_{A} ^{B}}
\end{aligned}
$$

Utilizing $s_{v, A P}=-s_{v, P B}$ again, it is possible to simplify $\overline{\tau_{t, A}}$ to:

$$
\overline{\tau_{t, A}}=-\mu\left(v_{m e s h}\right)\left|F_{n}\right| s_{v, A P} \frac{\int_{A}^{P} d_{A} \mathrm{~d} d_{A}-\int_{P}^{B} d_{A} \mathrm{~d} d_{A}}{\left.d_{A}\right|_{A} ^{B}} .
$$

Working out the integrals yield:

$$
\begin{aligned}
\overline{\tau_{t, A}} & =-\mu\left(v_{\text {mesh }}\right)\left|F_{n}\right| s_{v, A P} \frac{\left.d_{A}^{2}\right|_{A} ^{P}-\left.d_{A}^{2}\right|_{P} ^{B}}{\left.2 d_{A}\right|_{A} ^{B}}, \\
\overline{\tau_{t, A}} & =-\mu\left(v_{\text {mesh }}\right)\left|F_{n}\right| s_{v, A P} \frac{2{\overline{T_{1} P}}^{2}-{\overline{T_{1} A}}^{2}-{\overline{T_{1} B}}^{2}}{2\left(\overline{T_{1} B}-\overline{T_{1} A}\right)} .
\end{aligned}
$$

Again, in this equation the notation $\overline{T_{1} A}$ means the distance between point $T_{1}$ and point $A$. Since $\overline{T_{1} A}, \overline{T_{1} B}$ and $\overline{T_{1} P}$

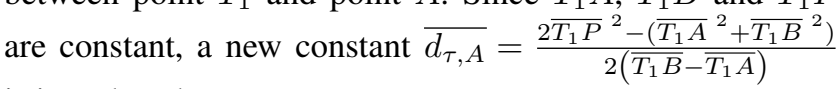
is introduced:

$$
\overline{\tau_{t, A}}=-\mu\left(v_{m e s h}\right)\left|F_{n}\right| s_{v, A P} \overline{d_{\tau, A}} \text {. }
$$

The constant $\overline{d_{\tau, A}}$ can be interpreted as an average lever arm on which the tangential force $F_{t}$ acts.
Calculation of $\overline{\tau_{t, B}}$ : The average $\overline{\tau_{t, B}}$ is calculated analog to the calculation of $\overline{\tau_{t, A}}$ :

$$
\begin{aligned}
\overline{\tau_{t, B}} & =\frac{\int_{A}^{B}-F_{t} d_{B} \mathrm{~d} d_{B}}{\int_{A}^{B} \mathrm{~d} d_{B}} \\
& =\frac{\int_{A}^{B}-\mu\left(v_{m e s h}\right) s_{v}\left(v_{m e s h}, d_{A}\right)\left|F_{n}\right| d_{B} \mathrm{~d} d_{B}}{\int_{A}^{B} \mathrm{~d} d_{B}} .
\end{aligned}
$$

Working out these integrals leads to:

$$
\overline{\tau_{t, B}}=-\mu\left(v_{m e s h}\right)\left|F_{n}\right| s_{v, A P} \frac{2{\overline{T_{2} P}}^{2}-{\overline{T_{2} A}}^{2}-{\overline{T_{2} B}}^{2}}{2\left(\overline{T_{2} B}-\overline{T_{2} A}\right)} .
$$

Since $\overline{T_{2} A}, \overline{T_{2} B}$ and $\overline{T_{2} P}$ are constant, a new constant $\left.\overline{d_{\tau, B}}=\frac{2{\overline{T_{2} P}}^{2}-\left({\overline{T_{2} A}}^{2}+\bar{T}_{2} B\right.}{2}\right)$ is introduced:

$$
\overline{\tau_{t, B}}=-\mu\left(v_{m e s h}\right)\left|F_{n}\right| s_{v, A P} \overline{d_{\tau, B}} .
$$

$\overline{d_{\tau, A}}$ and $\overline{d_{\tau, B}}$ contain the geometric information of the gear needed for the friction calculations. These values are purely defined by the gear geometry and are constant.

\section{Determination of the friction forces and moments in the case of $v_{\text {mesh }}=0$}

The static friction coefficient for $v_{\text {mesh }}=0$ is defined as $\mu_{0}$. Dependent on the position of the gear, the friction at zero speed can be zero (at rest with a single contact at the pitch point $P$, leading to $v_{s}=0$ ) or $\mu_{0}\left|F_{n}\right|$. Since no contact finding algorithms are used, the exact position of the gears is not known. It is therefore assumed that the contact is not at the pitch point and therefore not zero. Equation (15) can therefore be written as:

$$
F_{t}=\left\{\begin{array}{c}
\text { if } v_{\text {mesh }} \neq 0 \text { or }\left|F_{t}\right| \geq\left|\mu\left(v_{\text {mesh }}\right) F_{n}\right|: \\
s_{v} \mu\left(v_{\text {mesh }}\right)\left|F_{n}\right| \\
\text { if } v_{\text {mesh }}=0 \text { and }\left|F_{t}\right|<\left|\mu_{0} F_{n}\right|: \\
\text { so that } v_{\text {mesh }}=0
\end{array}\right.
$$

Note that $\mu_{0}$ (with $v_{\text {mesh }}=0$ ) is not necessarily the same as $\mu_{v_{\text {mesh }}}$ with $v_{\text {mesh }} \rightarrow 0$. This makes it possible to include static friction into the gear contact model using a state machine leading to a numerical efficient model. Using Equation (32), the friction force and moments for $v_{\text {mesh }}=0$ is given by:

$$
\left.\begin{array}{l}
\overline{F_{t}}=\mu_{0}\left|F_{n}\right| \overline{d_{F}} \\
\overline{\tau_{t, A}}=\mu_{0}\left|F_{n}\right| \overline{d_{\tau, A}} \\
\overline{\tau_{t, B}}=\mu_{0}\left|F_{n}\right| \overline{d_{\tau, B}}
\end{array}\right\} \text { for } \quad v_{m e s h}=0
$$

\section{Determination of the friction forces and moments}

In Table 2 the friction forces and moments are shown for each operational mode. This is done by substituting $s_{v, A P}$ in 
Table 2. Operational modes of the Gearbox. $Q$ is the operational quadrant.

\begin{tabular}{c|ccccccc}
\hline $\mathrm{Q}$ & $v_{\text {mesh }}$ & Power flow & $F_{n}$ & $\phi_{\text {contact }}$ & $\overline{F_{t}}$ & $\overline{\tau_{t, A}}$ & $\overline{\tau_{t, B}}$ \\
\hline 0 & 0 & - & - & - & $\mu_{0}\left|F_{n}\right| \overline{d_{F}}$ & $\mu_{0}\left|F_{n}\right| \overline{d_{\tau, A}}$ & $\mu_{0}\left|F_{n}\right| \overline{d_{\tau, B}}$ \\
1 & $>0$ & $A \rightarrow B$ & $>0$ & $<0$ & $\mu\left(v_{\text {mesh }}\right)\left|F_{n}\right| \overline{d_{F}}$ & $\mu\left(v_{\text {mesh }}\right)\left|F_{n}\right| \overline{d_{\tau, A}}$ & $\mu\left(v_{\text {mesh }}\right)\left|F_{n}\right| \overline{d_{\tau, B}}$ \\
2 & $>0$ & $B \rightarrow A$ & $<0$ & $>0$ & $-\mu\left(v_{\text {mesh }}\right)\left|F_{n}\right| \overline{d_{F}}$ & $\mu\left(v_{\text {mesh }}\right)\left|F_{n}\right| \bar{d}_{\tau, A}$ & $\mu\left(v_{\text {mesh }}\right)\left|F_{n}\right| \bar{d}_{\tau, B}$ \\
3 & $<0$ & $B \rightarrow A$ & $>0$ & $<0$ & $-\mu\left(v_{\text {mesh }}\right)\left|F_{n}\right| \overline{d_{F}}$ & $-\mu\left(v_{\text {mesh }}\right)\left|F_{n}\right| \overline{d_{\tau, A}}$ & $-\mu\left(v_{\text {mesh }}\right)\left|F_{n}\right| \overline{d_{\tau, B}}$ \\
4 & $<0$ & $A \rightarrow B$ & $<0$ & $>0$ & $\mu\left(v_{\text {mesh }}\right)\left|F_{n}\right| \bar{d}_{F}$ & $-\mu\left(v_{\text {mesh }}\right)\left|F_{n}\right| \overline{d_{\tau, A}}$ & $-\mu\left(v_{\text {mesh }}\right)\left|F_{n}\right| \overline{d_{\tau, B}}$ \\
\hline
\end{tabular}

Equation (22). For Equation (28) and (31), the contact angle $\phi_{\text {contact }}$ has to be considered next to $s_{v, A P}$ (see Figure 4 and 5). For $\phi_{\text {contact }}<0$ this leads to an extra multiplication with -1 to incorporate the correct direction of the average moments.

By analyzing Table 2 it becomes clear that the tangential part of the moment $\overline{\tau_{t}}$ and the friction force $\overline{F_{t}}$ are based on geometric gear constants, the mesh velocity, the friction coefficient and the normal force. To determine the mesh parameters from an unknown gear geometry, a good approximation can be made based on experience.

\section{Friction models}

To obtain the friction using the calculations from last sections, the friction coefficient $\mu\left(v_{\text {mesh }}\right)$ must be known. The friction coefficient can be calculated with different friction models and integrated in the gear model. In Assumption 2, the friction force is assumed to be constant over the line of action, and only dependent on the mesh velocity $v_{\text {mesh }}$. A modular approach is used for the implementation of the friction models which makes it possible to easily select the needed friction model. Following friction models are presented in the sections below:

1. Coulomb friction,

2. Friction model according to DIN3990-4,

3. Calculation of the friction coefficient from tabulated efficiency.

Depending on the purpose for which the model is used, a suitable friction model can be selected. The tabulated efficiency is not a real friction model, but allows the user to prescribe a speed dependent efficiency.

Coulomb friction: The Coulomb friction model is a simple friction model which is often used, when insufficient data is available. The Coulomb friction applies:

$$
\mu\left(v_{\text {mesh }}\right)=\left\{\begin{array}{lll}
\mu & \text { if } & \left|v_{\text {mesh }}\right|>0 \\
\mu_{0} & \text { if } & v_{\text {mesh }}=0
\end{array} .\right.
$$

In this model, a different friction coefficient for the stuck phase $\left(v_{\text {mesh }}=0\right)$ can be selected. This can be used to model a higher breakout force. To model a physical system, the static friction coefficient $\mu_{0}$ should be chosen such that: $\mu_{0} \geq \mu$. This also avoids numerical chattering around $v_{\text {mesh }}=0$.
Friction estimation from DIN3990-4: Norm DIN3990-4 ${ }^{4}$ proposes a friction model which depends on the mesh speed. This model bases on the dynamic oil viscosity $\eta_{\text {oil }}$, as well as on the loading of the gear. Its nonlinear friction characteristics can be used to estimate the gear friction for gear design based on design parameters.

$$
\mu\left(v_{\text {mesh }}\right)=\left\{\begin{array}{l}
\text { if }\left|v_{\text {mesh }}\right|>0: \\
0.12\left(\frac{w_{B t}}{\eta_{\text {oil }} v_{\sum C}}\right)^{0.25}\left(\frac{R_{a}}{\sigma_{\text {redC }}}\right)^{0.25} \\
\text { if } v_{\text {mesh }}=0: \\
\min \left(\mu_{0}, 0.12\left(\frac{w_{B t}}{\eta_{\text {oil }} v_{\sum C}}\right)^{0.25}\left(\frac{R_{a}}{\sigma_{\text {redC }}}\right)^{0.25}\right)
\end{array}\right.
$$

In Equation (35), $w_{B t}$ is the force per toothwidth, $R_{a}$ the mean roughness of the gear faces. Furthermore, the sum of the tangential velocities $\left(v_{\sum C}\right)$ at the pitch point $P$ is given by:

$$
v_{\sum C}=2\left|v_{m e s h}\right| \sin \left(\alpha_{w t}\right) .
$$

Here is $\alpha_{w t}$ the pressure angle of the gear. $\sigma_{r e d C}$ is the effective radius of curvature and is given by:

$$
\sigma_{r e d C}=1 e 3 \frac{i}{(1+i)^{2}} \frac{a \sin \left(\alpha_{w t}\right)}{\cos \left(\beta_{b}\right)},
$$

where $\beta_{b}$ is the helix angle, $i$ the gear ratio and $a$ the shaft center distance.

Tabulated efficiency: In the design process of aircraft actuators, usually experimental measurement results or estimates based on experiments are used. The design of controllers for such actuators is critical at low velocities and low temperatures where friction is high. For this condition however, hardly any theoretical results to predict the friction coefficient are valid. Therefore usually a tabulated gear efficiency is used. To be able to integrate this into the model, the efficiency must be expressed in the form of a friction coefficient which yields the set efficiency. Using symbolic manipulations with the symbolic toolbox from Matlab it is possible to express the friction coefficient $\mu\left(v_{\text {mesh }}\right)$ as a function of the total mesh velocity from $\eta=-\frac{\tau_{A} \omega_{A}}{\tau_{B} \omega_{B}}$ for 
quadrant $1 \& 4$, and $\eta=-\frac{\tau_{B} \omega_{B}}{\tau_{A} \omega_{A}}$ for quadrant $2 \& 3$.

$$
\mu\left(v_{\text {mesh }}\right)=\left\{\begin{array}{l}
\text { for quadrant } 1 \text { and } 4: \\
\left|\frac{r_{a} r_{b} \cos \left(\phi_{\text {contact }}\right)\left(\eta\left(v_{\text {mesh }}\right)-1\right)}{\overline{\tau_{t, B}} r_{a}-\overline{\tau_{t, A}} \eta\left(v_{\text {mesh }}\right) r_{b}}\right| \\
\text { for quadrant } 2 \text { and } 3: \\
\left|\frac{r_{a} r_{b} \cos \left(\phi_{\text {contact }}\right)\left(\eta\left(v_{\text {mesh }}\right)-1\right)}{\overline{\tau_{t, A}} r_{b}-\overline{\tau_{t, B}} \eta\left(v_{\text {mesh }}\right) r_{a}}\right|
\end{array} .\right.
$$

In this equation, $\eta\left(v_{m e s h}\right)$ is the tabulated efficiency. The static friction coefficient at $v_{m e s h}=0$ is given by the maximum of the friction coefficients:

$$
\begin{aligned}
\mu_{0}=c_{\mu_{0}} \max & \left(\left|\frac{r_{a} r_{b} \cos \left(\phi_{\text {contact }}\right)\left(\eta\left(v_{\text {mesh }}\right)-1\right)}{\overline{\tau_{t, B}} r_{a}-\overline{\tau_{t, A}} \eta\left(v_{\text {mesh }}\right) r_{b}}\right|,\right. \\
& \left.\left|\frac{r_{a} r_{b} \cos \left(\phi_{\text {contact }}\right)\left(\eta_{0}-1\right)}{\overline{\tau_{t, A}} r_{b}-\overline{\tau_{t, B}} \eta_{0} r_{a}}\right|\right)
\end{aligned}
$$

$c_{\mu_{0}} \geq 1$ is a constant which scales the efficiency in the stuck phase. It can be used to simulate a breakout friction with a friction coefficient higher than the friction at $v_{\text {mesh }} \rightarrow 0$. When $c_{\mu_{0}}=1$, the friction coefficient at $v_{m e s h}=0$ is the same as $v_{\text {mesh }} \rightarrow 0$.

\section{Gear stiffness and damping}

To model the gear stiffness and damping, several elasticity models are available. These spring models can be used for different simulations. These models range from a constant stiffness model for early design and simple modeling up to nonlinear stiffness with backlash, which can be used e.g. for rattling analysis of automotive gearboxes.

The proposed elasticity characteristics are implemented using an object oriented approach similar to the friction models from last section. This makes it possible to adjust the model to the simulation requirements. Furthermore, literature sources are not consistent on how the tooth stiffness varies with the load (e.g. ISO 6336-1 ${ }^{28}$ and Niemann $\&$ Winter $^{1}$ define the nonlinear spring characteristics differently). The modular approach chosen for the modeling of the gear contact can easily accommodate these different methods by supplying different spring characteristics. All models define $F_{n}$ as a function of $\Delta_{A B}$, the mesh deformation. In all models, a viscous damping is used. In Figure 7, an overview of the different elasticity characteristics is presented where the stiffness is defined as $\frac{\delta N}{\delta m}$. This is the elasticity when the model is linearized in a certain operation point. To be concise, only a selection of the stiffness models from 7 is presented in the next Sections. The other models can be obtained similarly.

Linear spring and damper: A simple stiffness model is provided for standard analysis with linear spring and damper characteristics. The normal force $F_{n}$ is defined by:

$$
F_{n}=c \Delta_{A B}+d \dot{\Delta}_{A B} .
$$

Nonlinear spring according to ISO 6336-1: Surface roughness $R_{q}{ }^{29}$ and tooth form deviations ${ }^{1}$ lead to

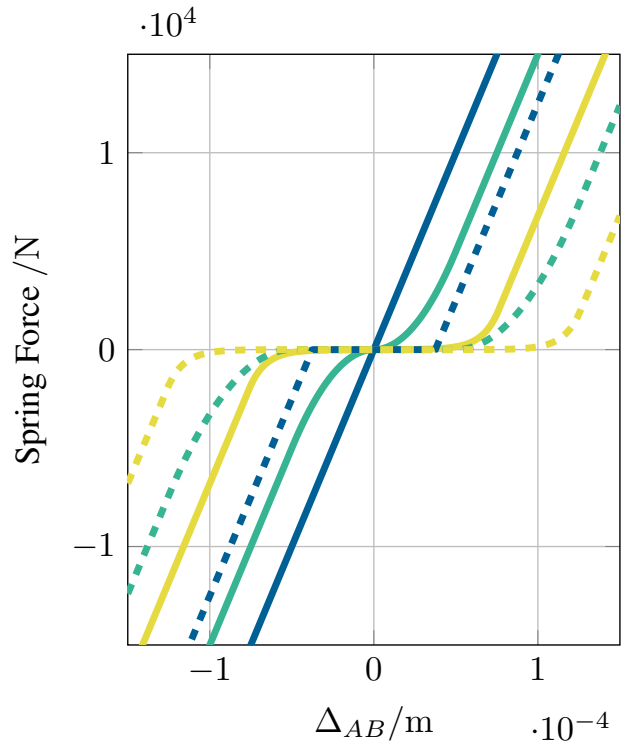

(a) Deformation of the contact

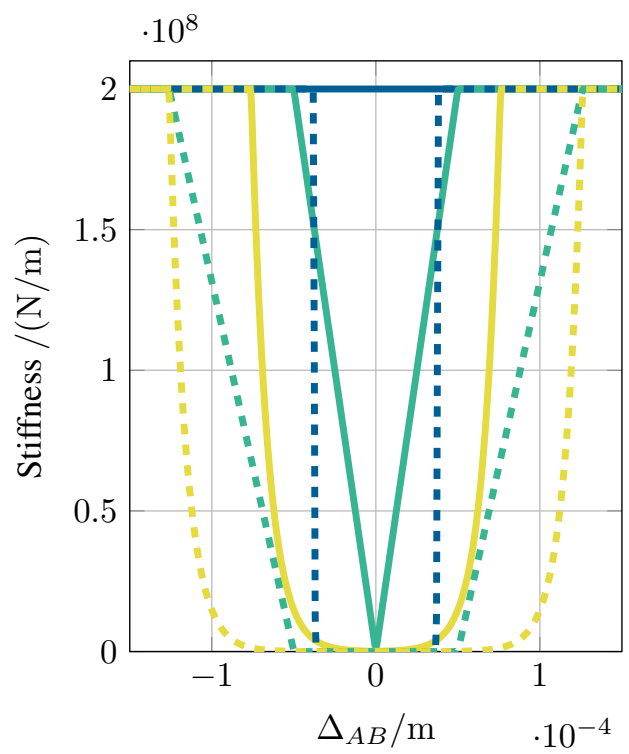

(b) Elasticity of the mesh

Figure 7. Characteristics of the different elasticity models:

1 is a linear elasticity model, = - - a model with backlash, a progressive elasticity model to ISO 6336-1 (- = - ISO 6336-1 model with backlash), a progressive elasticity model from Niemann and Winter $(=-m$ Niemann and Winter model with backlash).

a progressive tooth stiffness. The ISO 6336-1 norm ${ }^{28}$ proposes a progressive stiffness increasing linearly with the displacement. The maximal stiffness is reached at $\Delta_{A B}=$ $R_{q}$ where $R_{q}$ represents the surface roughness of the gears 
in contact. The normal force is given by:

$$
F_{n}=\left\{\begin{array}{c}
\text { if }\left|\Delta_{A B}\right|<R_{q}: \\
\frac{c}{2 R_{q}} \Delta_{A B}{ }^{2} \operatorname{sgn}\left(\Delta_{A B}\right)+d \dot{\Delta}_{A B} \\
\text { if }\left|\Delta_{A B}\right| \geq R_{q}: \\
\quad c\left(-\frac{R_{q}}{2}+\left|\Delta_{A B}\right|\right) \operatorname{sgn}\left(\Delta_{A B}\right)+d \dot{\Delta}_{A B}
\end{array}\right.
$$

In this equations, $c$ is the nominal stiffness coefficient and $d$ the damping coefficient.

Backlash: To simulate backlash of the gears, a dead zone $b$ is defined, in which the gears cannot transmit force. When the dead zone is passed, a linear stiffness model is used. In Equation (42) the normal force for the backlash model is developed:

$$
F_{n}=\left\{\begin{array}{l}
\text { if } \Delta_{A B}<-\frac{b}{2}: \\
c\left(\Delta_{A B}+\frac{b}{2}\right)+d \dot{\Delta}_{A B} \\
\text { if } \Delta_{A B}>\frac{b}{2}: \\
c\left(\Delta_{A B}-\frac{b}{2}\right)+d \dot{\Delta}_{A B} \\
\text { else: } 0
\end{array}\right.
$$

Backlash with nonlinear spring: The model of the Nonlinear spring according to ISO 6336-1 and the Backlash model can be combined in a single model with backlash and stiffness. The normal force $F_{n}$ is defined for this case by:

$$
F_{n}=\left\{\begin{array}{l}
\text { if } \Delta_{A B} \leq-\frac{b}{2}-R_{q}: \\
c\left(-\frac{R_{q}}{2}+\left|\Delta_{A B}+\frac{b}{2}\right|\right) \operatorname{sgn}\left(\Delta_{A B}\right)+d \dot{\Delta}_{A B} \\
\text { if }-\frac{b}{2}-R_{q}<\Delta_{A B}<-\frac{b}{2}: \\
\frac{c}{2 R_{q}}\left(\Delta_{A B}+\frac{b}{2}\right)^{2} \operatorname{sgn}\left(\Delta_{A B}\right)+d \dot{\Delta}_{A B} \\
\text { if }-\frac{b}{2} \leq \Delta_{A B} \leq \frac{b}{2}: \\
0 \\
\text { if } \frac{b}{2}<\Delta_{A B}<\frac{b}{2}+R_{q}: \\
\frac{c}{2 R_{q}}\left(\Delta_{A B}-\frac{b}{2}\right)^{2} \operatorname{sgn}\left(\Delta_{A B}\right)+d \dot{\Delta}_{A B} \\
\text { if } \Delta_{A B} \geq \frac{b}{2}+R_{q}: \\
c\left(-\frac{R_{q}}{2}+\left|\Delta_{A B}-\frac{b}{2}\right|\right) \operatorname{sgn}\left(\Delta_{A B}\right)+d \dot{\Delta}_{A B}
\end{array}\right.
$$

\section{Example: Modeling a spring loaded actuator}

In this Section, a simple spring loaded actuator is modeled. The behavior of the gear contact under preload has been investigated. It highlights the need for a gear model which can represent the behavior in a sticking phase.

\section{Model implementation}

An existing Modelica model of a gear contact without friction is presented in a previous publication ${ }^{3}$. In this work the model has been extended with an object-oriented implementation of friction and stiffness as presented in last Sections. A hybrid modeling approach, presented by Otter et al. ${ }^{30}$, is used to implement the friction including stiction as described in Table 2. This hybrid method calculates in quadrant 1 to 4 the friction force $F_{T}$ from the normal Force $F_{n}$ and friction coefficient $\mu\left(v_{\text {mesh }}\right)$. In quadrant 0 , a "stuck" mode is entered in which a different set of equations is used; Here the friction force is calculated from the force to keep the model from moving. For more detailed information see Otter et al. ${ }^{30}$.

Due to the correct friction handling at zero velocity as presented before, the model makes it possible to accurately model transmissions during normal continuous operation as well as low speed positioning- and position holding applications.

The PlanarMechanics library (see Zimmer ${ }^{31}$ ) is used to model the gear constraints and supply the model interfaces.

\section{Preloaded actuator sticking}

To show the functionality of the proposed model, gear sticking under preload is used to showcase the sticking behavior of the gear model. Ahmed ${ }^{14}$ and Garcia ${ }^{15}$ have shown the influence of stiction in geared actuators. In these actuators, the gears are often pre-loaded. This preload can originate from an aerodynamic load in the case of aircraft actuators or a pre-loading spring as used for example in geared air path actuators ${ }^{14}$. In the following example, a simplified model of such a spring loaded geared actuator is presented. The pre-load is realized in this example with a nonzero loading of gear $A$. This mimics a prestressed spring of a spring loaded actuator. In Figure 8 an overview of the combined model is shown. It consists of two elements to define the position of the bearings ( $\mathrm{fixed} \_$A and fixed_B). Two actuated revolute joints are shown as well: revolute_A is driven by a constant torque ramp to mimic an electrical motor with a current ramp, and revolute_B is connected to a spring damper system to mimic the pre-loaded spring. Also two bodies, Gear_A and Gear_B are used to model the gear mass and inertia. The gear element is the gear contact element proposed in this paper, using the DIN3990-4 friction model combined with the linear spring model. The tabulated and measured parameters of the gearbox are presented in Table 3, the fitted parameters in Table 4 . The parameters of a real gear contact are used, which will be used for the validation of the gear model.

In the gear simulation, the gear is preloaded with $5 \mathrm{Nm}$ and a ramp with $1 \mathrm{Nm} / \mathrm{s}$ is applied. The results are shown in Figure 9. As can be seen from this Figure, the model enters a stick-slip state, where the model sticks most of the 


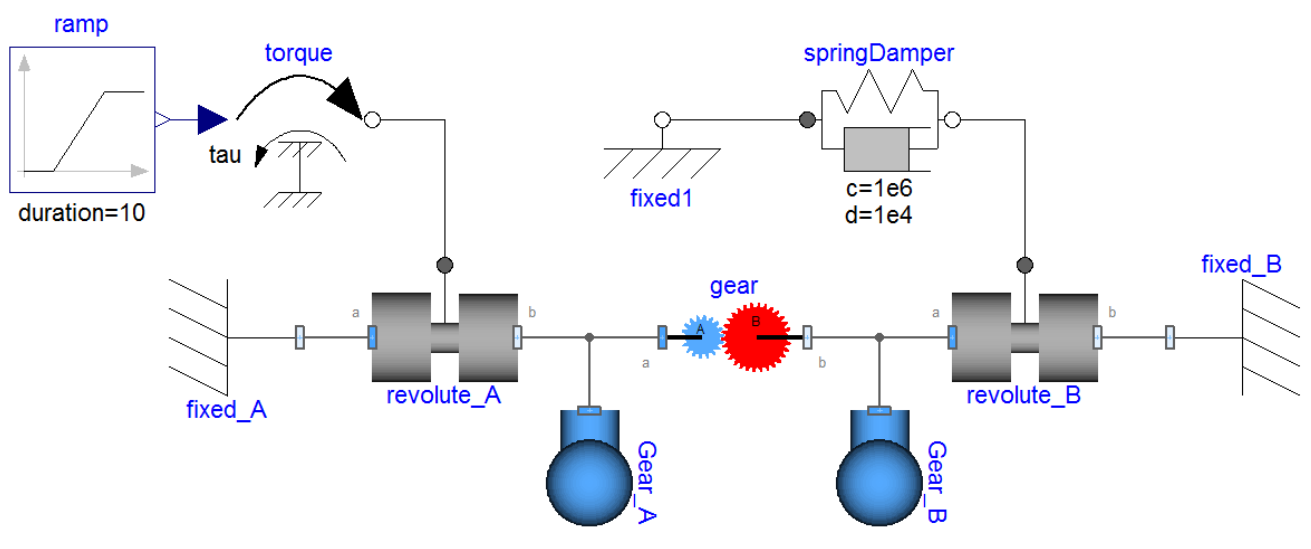

Figure 8. Gear model setup for sticking analysis

Table 3. Measured or tabulated Gearbox properties for the gearing examples

\begin{tabular}{|c|c|c|}
\hline $\begin{array}{l}\text { Gearbox } \\
\text { parameter }\end{array}$ & value & description \\
\hline$r_{A}$ & $10 \mathrm{~mm}$ & $\begin{array}{l}\text { Radius of gear } A \text { (pitch } \\
\text { radius). }\end{array}$ \\
\hline$r_{B}$ & $30 \mathrm{~mm}$ & $\begin{array}{l}\text { Radius of gear } B \text { (pitch } \\
\text { radius). }\end{array}$ \\
\hline$\phi_{\text {contact }}$ & $20^{\circ}$ & Contact angle. \\
\hline$m$ & $1 \mathrm{~mm}$ & Gear modulus. \\
\hline$r_{a A}$ & $11 \mathrm{~mm}$ & $\begin{array}{l}\text { Radius of the addendum } \\
\text { circle of gear } A \text {. }\end{array}$ \\
\hline$r_{a B}$ & $31 \mathrm{~mm}$ & $\begin{array}{l}\text { Radius of the addendum } \\
\text { circle of gear } B \text {. }\end{array}$ \\
\hline$\overline{d_{F}}$ & 0.84 & $\begin{array}{l}\text { Average friction force scal- } \\
\text { ing factor (Calculated from } \\
\text { the gear geometry). }\end{array}$ \\
\hline$\overline{d_{\tau, A}}$ & $-9.2 \mathrm{~mm}$ & $\begin{array}{l}\text { Average lever arm of } \tau_{A} \\
\text { (Calculated from the gear } \\
\text { geometry). }\end{array}$ \\
\hline$\overline{d_{\tau, B}}$ & $15.0 \mathrm{~mm}$ & $\begin{array}{l}\text { Average lever arm of } \tau_{B} \\
\text { (Calculated from the gear } \\
\text { geometry). }\end{array}$ \\
\hline$c$ & $2 \mathrm{E} 8 \mathrm{~N} / \mathrm{m}$ & Gear stiffness. \\
\hline$I_{A}$ & $5 \mathrm{E}-4 \mathrm{~kg} / \mathrm{m}^{2}$ & Inertia of gear $A$. \\
\hline$I_{B}$ & $1 \mathrm{E}-3 \mathrm{~kg} / \mathrm{m}^{2}$ & Inertia of gear $B$. \\
\hline$\eta_{o i l}$ & $275 \mathrm{Pas}$ & Dynamic oil viscosity \\
\hline
\end{tabular}

Table 4. Estimates and tuned valued for the gearing examples

\begin{tabular}{lll}
\hline $\begin{array}{l}\text { Gearbox } \\
\text { parameter }\end{array}$ & value & description \\
\hline$K_{B}$ & $28 \%$ & Effective tooth width. \\
$b$ & $15 \mu \mathrm{m}$ & Gear Backlash. \\
$d$ & $2 \mathrm{E} 5 \mathrm{~N} \mathrm{~s} / \mathrm{m}$ & Gear damping. \\
$R_{a}$ & $0.8 \mu \mathrm{m}$ & Gear roughness. \\
$\mu_{0}$ & 0.2 & Static friction coefficient. \\
\hline
\end{tabular}

time. When the breakout force is high enough, the model leaves the stuck phase, starts moving, to be stuck again shortly thereafter. The modeling approach described in Table 2 , guarantees that the mesh velocity stays zero in the stuck phase and no creepage due to imperfect handling of the friction at zero velocity is possible.

Note that the gear wheels $A$ and $B$ can still move at $v_{\text {mesh }}=0$. This movement is the elastic deformation of the gears. The overshoot in the position of gear $A$ is caused by the dynamic effect of the inertia of gear $A$ and the contact stiffness of the gear and springDamper element. When the gear contact comes in Forward mode, Gear $A$ increases its speed and a small overshoot is seen before the gear enters the Stuck mode again.

The computational costs of the simulation of the proposed system for $10 \mathrm{~s}$ (Figure 9) is $0.19 \mathrm{~s}$ computation time (Intel Xeon E5-1620, 16GB ram, DASSL solver, Dymola 2015). Using the proposed methods it is therefore possible to simulate a simple system approximately 50 times faster than real-time. This makes it possible to do optimization runs and long time simulations using the proposed gear contact model.

\section{Validation of the stiffness and friction models}

In this section, the presented gear contact model is validated against test rig results. This validation will be for selected friction- and stiffness models.

In a previous publication ${ }^{32}$, the gear test rig used in this paper has been presented. Using this test rig for spur gears (see Figure 10(b)), the elasticity and losses of the gear can be investigated. To do so, the same test rig has been modeled using the proposed gear models, combined with standard blocks from the Modelica standard library. The Modelica model of the testrig is presented in Figure 11. The losses are modeled according to DIN3990-4 friction model. As contact model, the backlash and nonlinear spring damper to Niemann \& Winter (Section 7(b)) has been selected.

\section{Gear contact analysis}

A gear contact of a gear transmission often has no perfect contact between the teeth. This non-perfect contact can be caused by misalignment of the gears and production imperfections of the gear wheels and shafts. In the gearbox from the test rig analyzed in this paper, the tooth contact pattern of the gears has been visualized using layout fluid. Running the gear at $80 \mathrm{~N}$ per one millimeter tooth width, the abrasion of the contact fluid shows the contact of the 


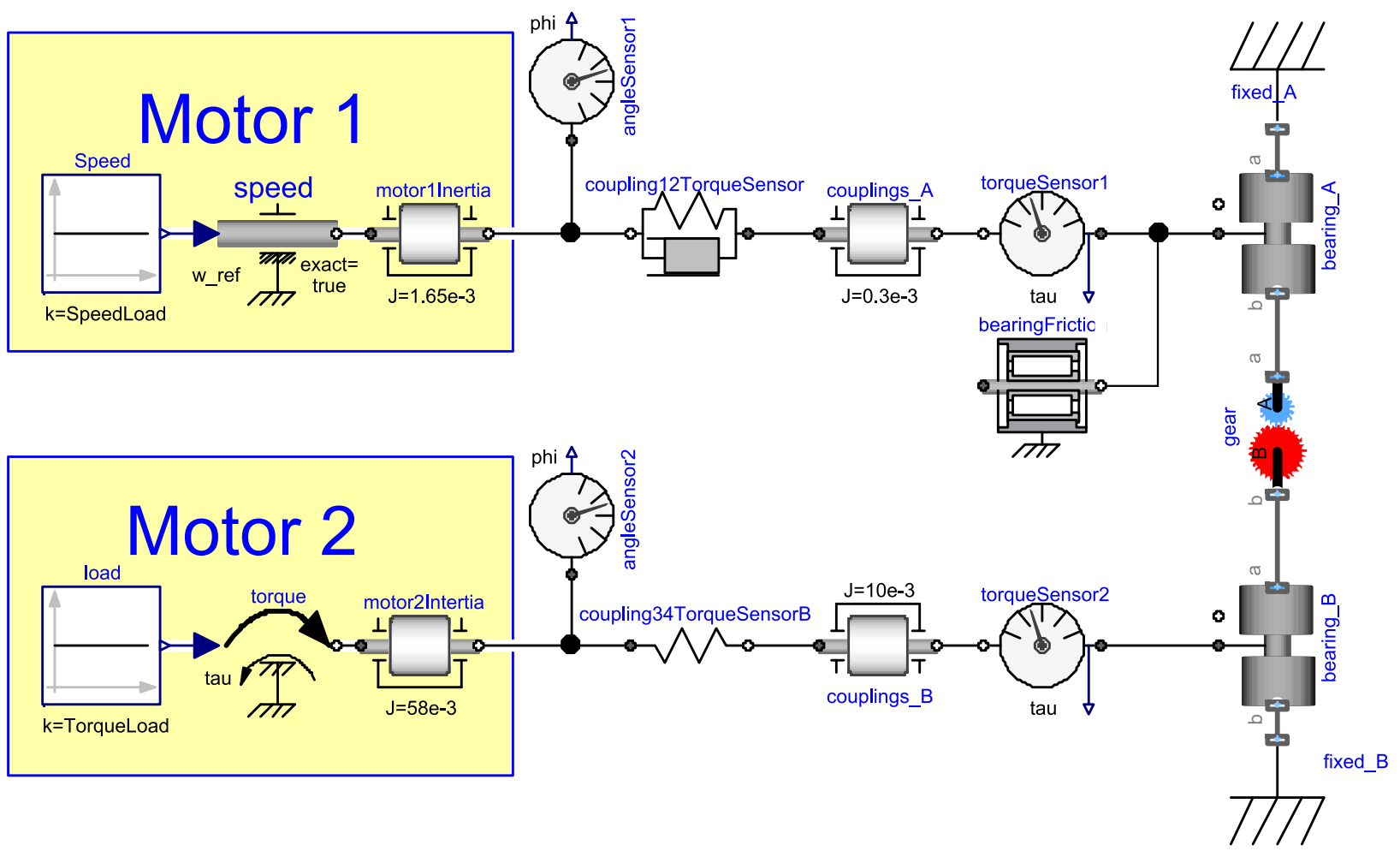

Figure 11. Testrig modeled in Dymola. Using this model the test results have been compared with the simulation results. In the model, the sensors, stiffness of the components and inertia of the masses have been taken into account.

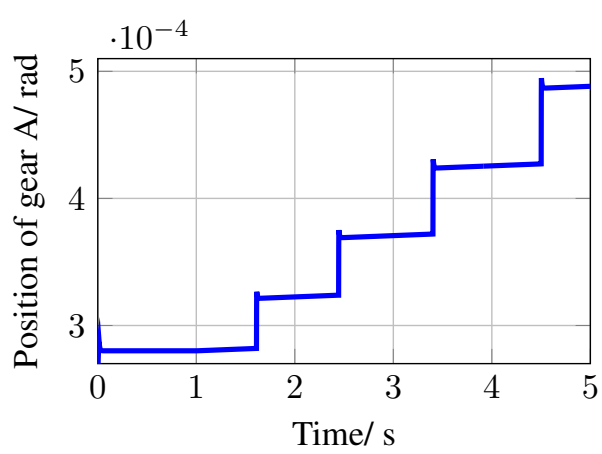

(a) Position of the gear during the loading of the gear

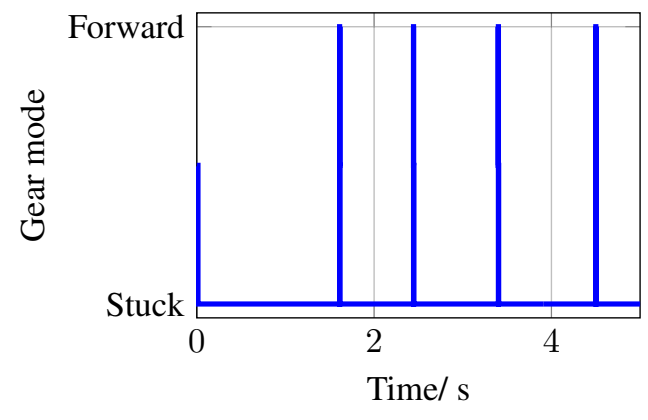

(b) Gear mode during the loading of the gear.

Figure 9. Gear sticking during the loading of a spring loaded gear. The shown stick slip phenomenon must be accurately handled for positioning actuators, since they can greatly influence the performance of the system.

gears as depicted in Figure 10(a). From the contact pattern it can be seen that not the complete gear face is in contact.

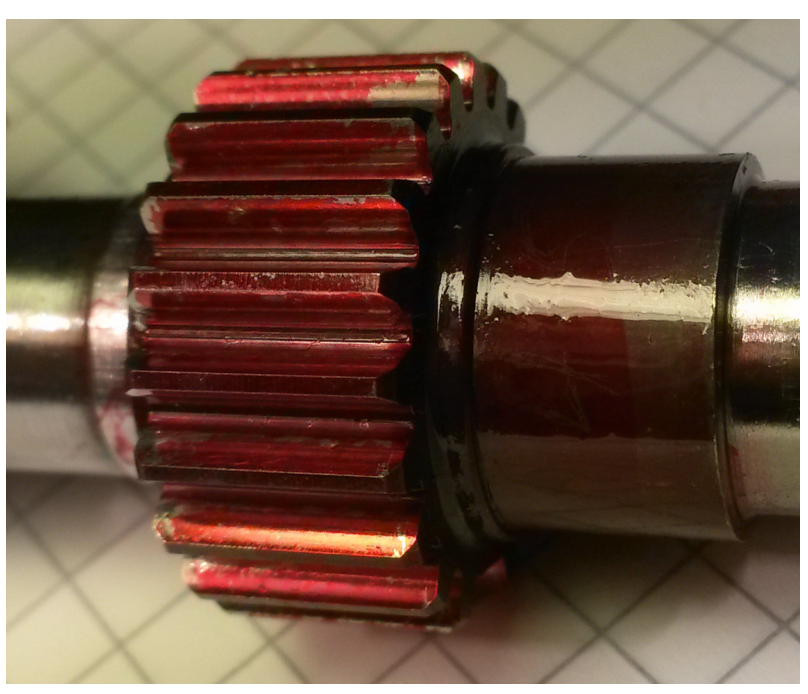

(a) Tooth contact pattern

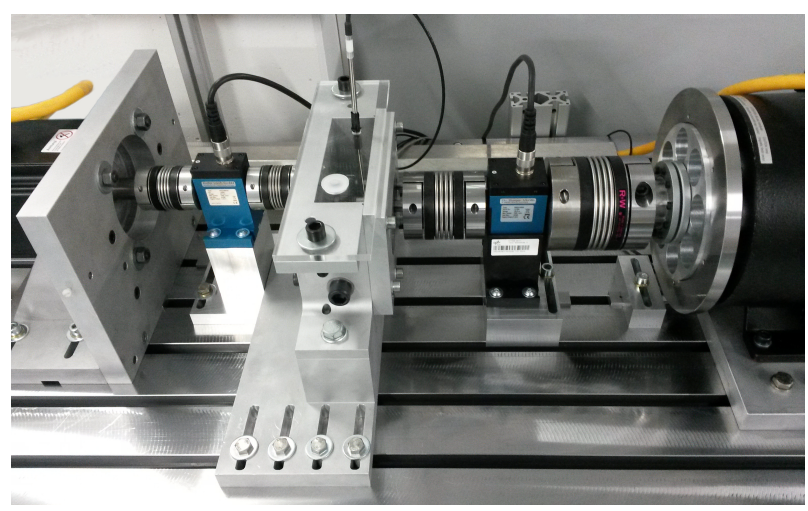

(b) Gear test rig

Figure 10. Gear test rig and contact pattern 
Accordingly the tooth width has been decreased to match the simulation.

\section{Mesh deformation and stiffness}

The mesh deformation and the stiffness of the proposed model are compared with the experimental testrig results in this section. To do so, the deformation $\Delta_{A B}$ (see Equation 9 ) is obtained as a function of the tooth loading $F_{n}$. This is done by stepwise increasing the load on the gear, while maintaining a constant low velocity of $\omega_{A}=10 \mathrm{rad} / \mathrm{s}\left(\mathrm{see}^{32}\right.$ for a detailed description). Note that $\phi_{A}$ and $\phi_{B}$ cannot be directly measured, since the position sensors are not directly at gear $A$ and $B$. To obtain the mesh deformation $\Delta_{A B}$, the stiffness of the gear train is taken into account:

$$
\Delta_{A B}=\Delta_{12}-F_{n} c_{\text {train }} .
$$

In this equation, $\Delta_{12}$ is the deformation between the sensor positions and $c_{\text {train }}$ the apparent stiffness of the train at the contact position including the stiffness of the couplings, sensors and axis. The stiffness is obtained using data-sheets of couplings and sensors as well as stiffness calculations based on the cross section of the axis. The stiffness of the gear contact is obtained by differentiation of the deformation of the gear with respect to the load. To avoid the influence of the rotational position of the gears like eccentricities and tooth effects, the average of the deformation and stiffness of all gear positions has been used.

To compare the presented models with the test rig, the test rig has been modeled including the train stiffness. The contact stiffness of the gear has been estimated to be $20 \mathrm{~N} / \mu \mathrm{m}$ per $1 \mathrm{~mm}$ tooth width as suggested by Niemann and Winter ${ }^{1}$ Chapter 21.5. The effective tooth width is identified to match the stiffness measured on the testrig. The effective tooth width has been optimized to fit the measurement data and is identified to be $28 \%$ of the original tooth width. This result can be matched with the tooth contact pattern as seen in Figure 10(a). The gear backlash is set to $15 \mu \mathrm{m}$ to match the measurement results. The lumped static friction of the seals and bearings of the gear has been measured to be $0.05 \mathrm{~N} / \mathrm{m}$. This is included in the Modelica model with a standard bearing model (bearingFriction in Figure 11) which acts as a constant, speed independent friction. An overview of the measured and tabulated parameters is given in Table 3, the fitted parameters in Table 4.

Mesh deformation and stiffness measurements: In Figure 12 , a comparison of the average relative displacement of the gear as well as the stiffness of the gear as a function of the contact load is shown. The solid lines show the measurement data, the dashed lines simulation data. In this figure, the gear has been run at positive and negative angular velocities to be able to show all 4 quadrants.

Stiffness validation: Comparing the measurement results with the simulation results, the gear contact deformation of the simulations shows a good fit with the measurement data (see Figure 12(a)). The maximal deviation between the simulation and measurement is $10 \mu \mathrm{m}$ with an average error of $3.4 \mu \mathrm{m}$. The comparison of the stiffness between model and simulation shows a larger deviation (Figure 12(b)).

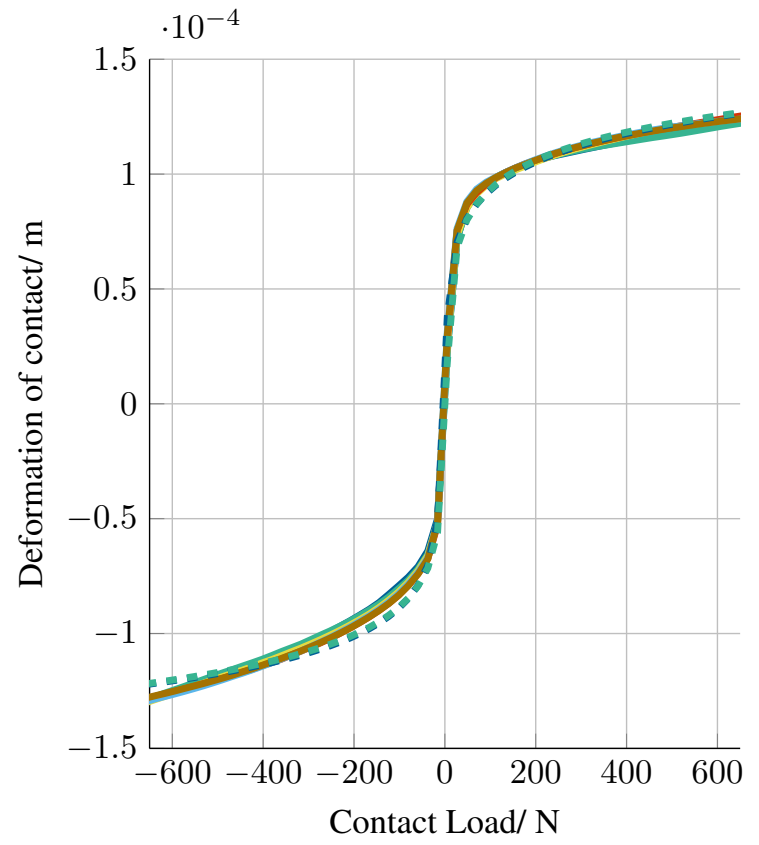

(a) Deformation of the gear contact

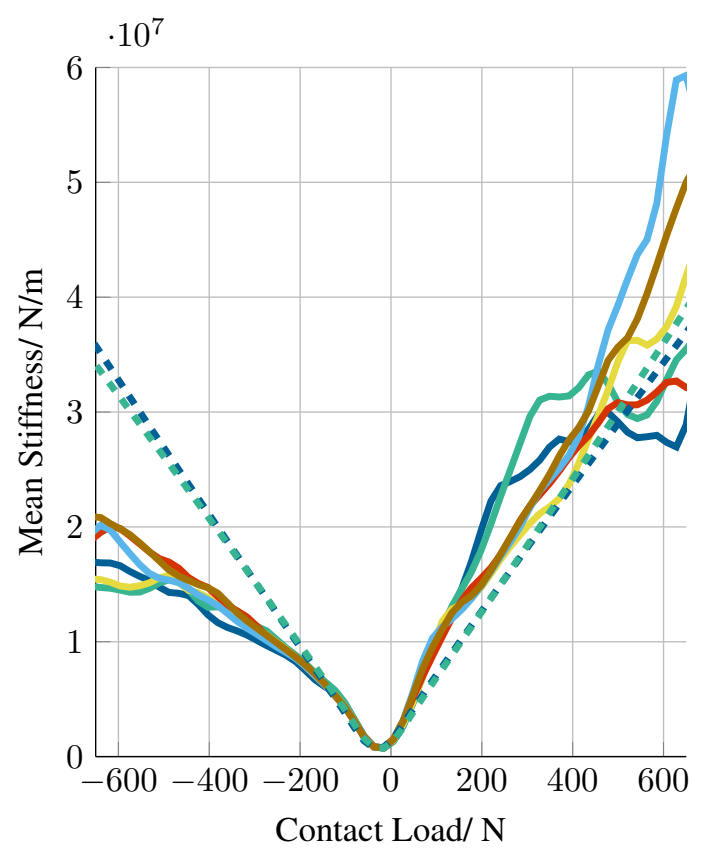

(b) Elasticity of the gear contact

Figure 12. The average deformation of the mesh and mesh stiffness of the gear as a function of the contact load. The results show two simulation results with opposite rotational velocity (dashed lines) as well as 6 measurements results (solid lines).

Looking at the measurement data, the stiffness increases linearly with the load, but is not symmetric: At positive loads a higher stiffness is observed than with negative loads. This can be caused by manufacturing imperfections like gear roughness and misalignment of the gears and axis. The stiffness of the simulation model also increases linear with the load. However, it cannot exactly predict the stiffness of the gear contact with a high accuracy. This is mainly due to the fact that the gear parameters with respect to manufacturing errors are not available. The gear stiffness 


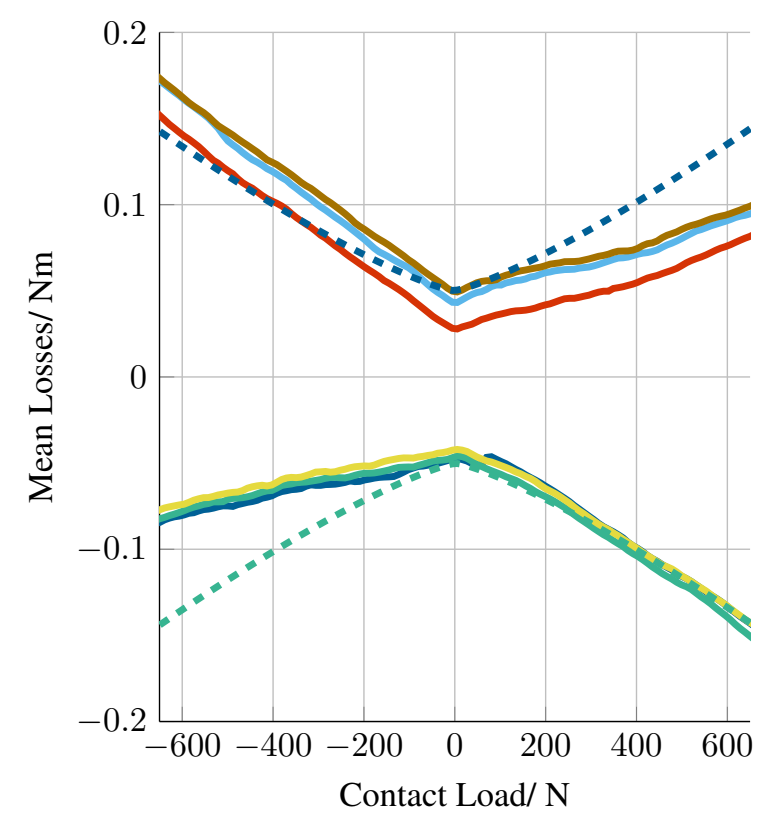

Figure 13. The average friction characteristics of the gear are shown as a function of the contact load. The dashed lines are simulation results with opposite rotational velocity, the solid lines are measurement results.

could be made dependent on the loading side of the gear, and a contact stiffness fitted to match the gear contact if required. However, this poses the question on how to obtain these data, and if this data is consistent between different gear contacts.

\section{Gear contact losses}

The gear contact losses are modeled using the approach from the DIN3990-4 Norm. The parameters for the gear friction model are the same as in the testrig: The oil viscosity is $320 \mathrm{~mm}^{2} / \mathrm{s}$. Again, the tooth width is set to $28 \%$ of the original tooth width, as identified before. The gear roughness is set to $0.8 \mu \mathrm{m}$. The mean losses of the gearbox can be estimated by:

$$
\tau_{\text {friction }}=\tau_{A}+\tau_{B} \frac{r_{A}}{r_{B}} .
$$

Note that the friction losses in Equation 45 are the combined friction losses of the gear plus bearings and seals since the test rig does not allow direct friction measurements without the bearings and seals. The friction estimated in Equation (45) is shown as a function of the contact load in Figure 13. The friction increases with the gear load and simulation results predict the gear losses accurately. However, also here can be seen that just like the gear stiffness, the friction of the gears is not symmetric. As discussed before, this asymmetry can be explained by manufacturing imperfections. The maximal deviation is $0.09 \mathrm{~N} \mathrm{~m}$ and the mean deviation of the friction is for all load cases $0.023 \mathrm{Nm}$. The measured noload friction is confirmed to be $0.5 \mathrm{Nm}$. Using the presented models it is possible to accurately represent the behavior of real life gear contacts if the gear parameters are well known.

\section{Conclusion and summary}

With the gear model presented in this article, it is possible to model a gear contact with involute teeth. Elastic effects as well as the contact friction are considered. The model ensures a good trade-off between simulation accuracy and good computational performance. This makes the model suitable for design and optimization of gear transmission integrated in complete systems like aircraft actuators. The treatment of the friction at zero velocity enables analysis with stiction and stick-slip cases. This enables the modeling of positioning systems as well as low speed applications like low temperature and therefore high friction environments such as electromechanical aircraft actuators. Due to the modular implementation, it is possible to use the presented gear contact without modification of the contact itself in different gearing configurations. Different friction models have been implemented, as well as different stiffness models to cater for different gearing scenarios. The load dependent friction and stiffness of the simulations have been validated using a dedicated gear testrig.

\section{Acknowledgements}

I thank D. J. Rixen and J. Tobolar for their valuable comments, which made this work better understandable.

This research received no specific grant from any funding agency in the public, commercial, or not-for-profit sectors.

\section{References}

1. Gustav Niemann and Hans Winter. Maschinenelemente: Band 2: Getriebe allgemein, Zahnradgetriebe - Grundlagen, Stirnradgetriebe (German Edition). Springer, 1989.

2. Earle Buckingham. Analytical mechanics of gears. McGrawHill, London, 1949.

3. Franciscus L. J. van der Linden. Modelling of Elastic Gearboxes Using a Generalized Gear Contact Model. In Proceedings of the 9th International MODELICA Conference, pages 303-310, Munich, November 2012. Linkoping University Electronic Press. doi:10.3384/ecp12076303.

4. DIN 3990 Teil 4. Tragfähigkeitsberechnung von Stirnrädern; Berechnung der Freßtragfähigkeit, 1987.

5. Chinmaya Kar and A. R. Mohanty. Vibration and current transient monitoring for gearbox fault detection using multiresolution Fourier transform. Journal of Sound and Vibration, 311(1-2):109-132, March 2008. doi:10.1016/ j.j.jv. 2007.08 .023$.

6. P Velex and V Cahouet. Experimental and Numerical Investigations on the Influence of Tooth Friction in Spur and Helical Gear Dynamics. Journal of Mechanical Design, 122(4):515, 2000. doi:10.1115/1.1320821.

7. P. Velex and M. Maatar. A mathematical model for analyzing the influence of shape deviations and mounting errors on gear dynamic behaviour. Journal of Sound and Vibration, 191(5):629-660, April 1996. doi:10.1006/jsvi.1996. 0148.

8. P. Velex and P. Sainsot. An analytical study of tooth friction excitations in errorless spur and helical gears. Mechanism and Machine Theory, 37(7):641-658, July 2002. doi : 10 . 1016/ S0094-114X (02) 00015-0.

9. P. Velex and F. Ville. An Analytical Approach to Tooth Friction Losses in Spur and Helical Gears - Influence of Profile Modifications. Journal of Mechanical Design, 131(10):101008, 2009. doi:10.1115/1.3179156. 
10. H Vinayak and R Singh. Multi-body dynamics and modal analysis of compliant gear bodies. Journal of Sound and Vibration, 210(2):171-214, February 1998. doi:10.1006/ jsvi.1997.1298.

11. H Vinayak, R Singh, and C Padmanabhan. Linear dynamic analysis of multi-mesh transmissions containing external, rigid gears. Journal of Sound and Vibration, 185(1):1-32, August 1995. doi:10.1006/jsvi.1994.0361.

12. Ahmet Kahraman and R Singh. Non-Linear Dynamic Analysis of Geared Systems. Journal of Sound and Vibration, 142(1):49-75, 1990. doi:10.1016/0022-460X(90) $90582-\mathrm{K}$.

13. James R. Ottewill, Simon a. Neild, and R. Eddie Wilson. Intermittent gear rattle due to interactions between forcing and manufacturing errors. Journal of Sound and Vibration, 321(35):913-935, April 2009. doi:10.1016/j.jsv.2008. 09.050 .

14. F. S. Ahmed, S. Laghrouche, and M. El Bagdouri. Overview of the modelling techniques of actuator non-linearities in the engine air path. Proceedings of the Institution of Mechanical Engineers, Part D: Journal of Automobile Engineering, 227(3):443-454, September 2012. doi:10. $1177 / 0954407012453905$.

15. Elena Garcia, PG de Santos, and CC de Wit. Velocity dependence in the cyclic friction arising with gears. The International Journal of Robotics Research, 21(9):761-771, 2002. doi:10.1177/0278364902021009877.

16. H. Nevzat Özgüven and D.R. Houser. Mathematical models used in gear dynamics, A review. Journal of Sound and Vibration, 121(3):383-411, March 1988. doi:10.1016/ s0022-460X (88) 80365-1.

17. A Parey and N Tandon. Spur Gear Dynamic Models Including Defects: A Review. The Shock and Vibration Digest, 35:465478, 2003. doi:DOI:10.1177/05831024030356002.

18. Yi Guo and Robert G Parker. Dynamic Analysis of Planetary Gears With Bearing Clearance. Journal of Computational and Nonlinear Dynamics, 7(4):41002, 2012. doi:10.1115/1. 4005929.

19. Robert G Parker and Xionghua Wu. Vibration modes of planetary gears with unequally spaced planets and an elastic ring gear. Journal of Sound and Vibration, 329(11):2265-2275, 2010. doi:10.1016/j.jsv.2009.12.023.

20. Rune Pedersen, Ilmar F Santos, and Ivan A Hede. Advantages and drawbacks of applying periodic time-variant modal analysis to spur gear dynamics. Mechanical Systems and Signal Processing, 24(5):1495-1508, 2010. doi:DOI:10.1016/ j.ymssp.2009.12.009.

21. Timo Kiekbusch, Daniel Sappok, Bernd Sauer, and Ian Howard. Calculation of the Combined Torsional Mesh Stiffness of Spur Gears with Two- and Three-Dimensional Parametrical FE Models. Strojniški vestnik - Journal of Mechanical Engineering, 57(11):810-818, November 2011. doi:10.5545/sv-jme.2010.248.

22. Manish Vaishya and Rajendra Singh. Strategies for Modeling Friction in Gear Dynamics. Journal of Mechanical Design, 125(2):383, 2003. doi:10.1115/1.1564063.

23. C Pelchen, C Schweiger, and M Otter. Modeling and Simulating the Efficiency of Gearboxes and of Planetary Gearboxes. In 2nd International Modelica Conference, volume 3, pages 257-266, 2002.
24. J. D. de Gevigney, F. Ville, C. Changenet, and P. Velex. Tooth friction losses in internal gears: Analytical formulation and applications to planetary gears. Proceedings of the Institution of Mechanical Engineers, Part J: Journal of Engineering Tribology, 227(5):476-485, January 2013. doi:10.1177/ 1350650112470059 .

25. Saeed Ebrahimi and Peter Eberhard. Rigid-elastic modeling of meshing gear wheels in multibody systems. Multibody System Dynamics, 16(1):55-71, July 2006. doi:10.1007/ s11044-006-9021-7.

26. Ullrich Kissling and $M$ Raabe. Calculating Tooth Form Transmission Error. gearsolutions.com, September:3966, 2006. URL: http://gearsolutions. com/article/detail/5442/title/

calculating-tooth-form-transmission-error.

27. Neil E Anderson and Stuart H Loewenthal. Spur-gear-system efficiency at part and full load. Technical Report 46, NASA, 1980.

28. ISO 6336-1. Calculation of load capacity of spur and helical gears, 2007.

29. Xi Shi and Andreas a. Polycarpou. Measurement and Modeling of Normal Contact Stiffness and Contact Damping at the Meso Scale. Journal of Vibration and Acoustics, 127(1):52-60, 2005. doi:10.1115/1.1857920.

30. M Otter, H Elmqvist, and S E Mattsson. Hybrid modeling in Modelica based on the synchronous data flow principle. In Computer Aided Control System Design, 1999. Proceedings of the 1999 IEEE International Symposium on, pages 151-157, 1999. doi:10.1109/CACSD.1999.808640.

31. Dirk Zimmer. A Planar Mechanical Library for Teaching Modelica. In Proceedings of the 9th International Modelica Conference, pages 681-690, Munich, November 2012. Linköping University Electronic Press. doi:10. 3384 / ecp12076681.

32. Franciscus Leendert Johannes van der Linden. Gear test rig for health monitoring and quasi static- and dynamic testing; design, construction and first results. In Proceedings of the International Gear Conference 2014, pages 976-985, Lyon, 2014. Woodhead Publishing. URL: http://elib.dlr. de/90564/. 